\title{
Largest $\boldsymbol{j}$-Simplices in $n$-Polytopes*
}

\author{
P. Gritzmann, ${ }^{1}$ V. Klee, ${ }^{2}$ and D. Larman $^{3}$ \\ ${ }^{1}$ Fb. IV, Mathematik, Universität Trier, \\ D-54286 Trier, Germany \\ gritzman@dm1.uni-trier.de \\ ${ }^{2}$ Department of Mathematics, GN-50, University of Washington, \\ Seattle, WA 98195, USA \\ klee@math.washington.edu \\ ${ }^{3}$ Department of Mathematics, University College, \\ London WC1E 6BT, England \\ ucahdgl@ucl.ac.uk
}

\begin{abstract}
Relative to a given convex body $C$, a $j$-simplex $S$ in $C$ is largest if it has maximum volume ( $j$-measure) among all $j$-simplices contained in $C$, and $S$ is stable (resp. rigid) if $\operatorname{vol}(S) \geq \operatorname{vol}\left(S^{\prime}\right)$ (resp. $\operatorname{vol}(S)>\operatorname{vol}\left(S^{\prime}\right)$ ) for each $j$-simplex $S^{\prime}$ that is obtained from $S$ by moving a single vertex of $S$ to a new position in $C$. This paper contains a variety of qualitative results that are related to the problems of finding a largest, a stable, or a rigid $j$-simplex in a given $n$-dimensional convex body or convex polytope. In particular, the computational complexity of these problems is studied both for $\mathscr{V}$-polytopes (presented as the convex hull of a finite set of points) and for $\mathscr{H}$-polytopes (presented as an intersection of finitely many halfspaces).
\end{abstract}

\section{Introduction}

The setting for everything in this paper is a finite-dimensional Euclidean space $\mathbb{R}^{n}$. As the terms are used here, a body in $\mathbb{R}^{n}$ is a compact convex set with nonempty interior and a polytope is a body that has only finitely many extreme points. Prefixes are often used to indicate dimension. For $1 \leq j \leq n$, a $j$-simplex is the convex hull of a set of $j+1$ affinely independent points. Relative to a given body $C$, a $j$-simplex $S$

*The research of the first and second authors was supported in part by the Deutsche Forschungsgemeinschaft and by a joint Max-Planck Research Award. The research of the second author was also supported in part by the Mittag-Leffler Institute and the National Science Foundation. 
in $C$ is largest if $S$ has maximum volume ( $j$-measure) among all $j$-simplices contained in $C$, and $S$ is stable (resp. rigid) if $\operatorname{vol}(S) \geq \operatorname{vol}\left(S^{\prime}\right)\left(\operatorname{resp} . \operatorname{vol}(S)>\operatorname{vol}\left(S^{\prime}\right)\right)$ for each $j$-simplex $S^{\prime}$ that is obtained from $S$ by moving a single vertex of $S$ to a new position in $C$. The simplex $S$ is bound to $C$ if each vertex of $S$ is an extreme point of $C$. These notions, or variants of them, occur throughout this paper.

The paper was motivated by a desire to gain a better understanding of the algorithmic difficulty of finding a largest $j$-simplex in a given high-dimensional polytope $P$. There is always a largest $j$-simplex that is bound, and hence when $P$ has $m$ vertices the number of volume computations needed to find a largest $j$-simplex in $P$ does not exceed $\left(\begin{array}{c}m \\ j+1\end{array}\right)$. However, that may be a very large number in cases of interest, and many challenges are provided even by the special case in which $j=n$ and $P$ is the unit $n$-cube. (That case is discussed in detail in a companion paper [HKL], and some applications are described in [GK4].) Also, since polytopes are among the most familiar of geometric objects and simplices are the simplest sort of polytope, it seems fair to claim that the problem of finding a largest $j$-simplex in a given $n$-polytope is a basic and prototypical problem in computational convexity. See [GK4] for a survey of related containment problems.

Here are our section headings: 1. Determinantal Tools; 2. Largest and Stable Simplices in Convex Bodies; 3. Moving a Bound Simplex in a Convex Polytope; 4. Computational Preliminaries, Tractability Results; 5. Hardness Results for $\mathscr{V}$-Polytopes; 6. Hardness Results for $\mathscr{H}$-Polytopes; and 7. Additional Comments and Problems.

\section{Determinantal Tools}

Throughout this paper there are close interactions between volumes and determinants. This section assembles some results involving determinants that are used in later sections. In Section 2 the following two results play an essential role in establishing relationships between the vertices of largest simplices and the facial structure of the containing body.

Theorem 1.1. Suppose that $A$ and $B$ are $n \times n$ matrices, that $A$ is invertible, and that the matrix $A+\operatorname{diag}\left(\tau_{1}, \ldots, \tau_{n}\right) B$ has the same determinant for all real $\tau_{1}, \ldots, \tau_{n}$. Then at least one row of $B$ is zero.

Proof. If satisfied by $(A, B)$, the hypotheses are also satisfied by the pair ( $A A^{-1}, B A^{-1}$ ). Since the conclusion for the latter pair implies that for the former, we may assume without loss of generality that $A=I$ and, with

$$
f\left(\tau_{1}, \ldots, \tau_{n}\right)=\operatorname{det}\left(I+\operatorname{diag}\left(\tau_{1}, \ldots, \tau_{n}\right) B\right),
$$

that $f \equiv 1$. Note that this assumption is hereditary in the sense that it is still satisfied when the $i$ th row and $i$ th column are deleted from each of $I, \operatorname{diag}\left(\tau_{1}, \ldots, \tau_{n}\right)$, and $B$; this corresponds to the choice $\tau_{i}=0$.

When $n=1$, it is obvious that $B$ has a zero row. We now consider a "smallest counterexample" in order to derive a contradiction. So suppose that the assertion 
fails for some $n \geq 2$ and $n \times n$ matrix $B$, but holds for each smaller $n$ and corresponding matrix.

The function $f$ is a polynomial in $\tau_{1}, \ldots, \tau_{n}$, and for each set of indices $N \subset\{1, \ldots, n\}$ the coefficient of the product $\Pi_{k \in N} \tau_{k}$ in $f$ is equal to $\operatorname{det}\left(B_{N}\right)$, where $B_{N}$ is obtained from the matrix $B=\left(\beta_{i j}\right)$ by deleting each row and column of $B$ whose index does not belong to $N$ (and agreeing that the product over the empty set and the determinant of the empty matrix are both equal to 1). Now consider an $i \in\{1, \ldots, n\}$ and let $N_{i}=\{1, \ldots, n\} \backslash\{i\}$. It follows from the minimality of $n$ that $B_{N_{1}}$ has a zero row with index (say) $\pi(i)$, but this row is not zero in $B$ and hence $\beta_{\pi(i) i} \neq 0$. Also, if $j \in\{1, \ldots, n\}$ and $j \neq i$, then $\pi(i) \neq \pi(j)$, for otherwise both $\beta_{\pi(i) i}$ and $\beta_{\pi(i) j}$ are nonzero in row $\pi(i)$ of $B$, contradicting the fact that the $\pi(i)$ row of $B_{N_{t}}$ is zero. We may now conclude that the matrix $B$ has exactly one nonzero entry in each row and each column, whence $\operatorname{det}(B) \neq 0$. However, $\operatorname{det}(B)$ is the coefficient of the product $\tau_{1} \cdots \tau_{n}$ in the equation

$$
\operatorname{det}\left(I+\operatorname{diag}\left(\tau_{1}, \ldots, \tau_{n}\right) B\right) \equiv 1,
$$

and if $\operatorname{det}(B) \neq 0$, then for $\tau_{1}=\tau_{2}=\cdots=\tau_{n}=\tau>0$ the left-hand side is dominated by the positive term $\tau^{n}$ for large $\tau$. From this contradiction it follows that at least one row of $B$ is zero.

Corollary 1.2. With hypotheses as in Theorem 1.1, suppose that $B$ has a nonzero row. Then at least one such row is a linear combination of the rows of $A$ that correspond to zero rows of $B$.

Proof. Suppose, with $1 \leq r<n$, that the first $r$ rows of $B$ are nonzero and the last $n-r$ rows of $B$ are zero. Then, starting with the matrix $I+\operatorname{diag}\left(\tau_{1}, \ldots, \tau_{n}\right) B$, we can subtract suitable multiples of the last $n-r$ rows from the first $r$ rows to obtain a new matrix in which all entries beyond the $r$ th column are zero in each of the first $r$ rows. The determinant of the new matrix is still independent of the choice of the $\tau_{i}$, and from this it follows that if $\hat{B}$ is the upper left $r \times r$ submatrix of $B$, then

$$
\operatorname{det}\left(I+\operatorname{diag}\left(\tau_{1}, \ldots, \tau_{r}\right) \hat{B}\right) \equiv 1 \text {. }
$$

By the result already proved, this implies that at least one row of $\hat{B}$ is zero and hence one of the first $r$ rows of $B$ is linearly dependent on the last $n-r$ rows of $A$.

The following facts about simplex volumes are very well known (see [S3]). They are used here without specific reference, or are referred to as "standard formulas":

- If $v$ is a vertex of a $j$-simplex $S, F$ is the facet ( $(j-1)$-face) of $S$ that misses $v$, and $\delta$ is the distance from $v$ to the affine hull aff $(F)$ of $S$, then $\operatorname{vol}(S)=$ $\delta \operatorname{vol}(F) / j$.

- If $S$ is an $n$-simplex in $\mathbb{R}^{n}$ and $A$ is the $(n+1) \times n$ matrix whose rows list the coordinates of the vertices of $S$, then $(n !) \operatorname{vol}(S)=|\operatorname{det}(M)|$, where $M$ is the $(n+1) \times(n+1)$ matrix formed from $A$ by appending a column of 1 's. If the origin is a vertex of $S$, then $(n !) \operatorname{vol}(S)=\left|\operatorname{det}\left(A_{0}\right)\right|$ where $A_{0}$ is formed from $A$ by discarding $A$ 's zero row. 
- The circumradius and the volume of a regular $j$-simplex of edge-length $\lambda$ are respectively equal to

$$
\lambda \sqrt{\frac{j}{2(j+1)}} \text { and } \frac{\sqrt{j+1}}{j !}\left(\frac{\lambda}{\sqrt{2}}\right)^{j} \text {. }
$$

Hence the volume of a regular $j$-simplex of circumradius $\rho$ is equal to

$$
\frac{(j+1)^{(j+1) / 2}}{j ! j^{j / 2}} \rho^{j} .
$$

We also use the fact, proved by Fejes Tóth [F, p. 313] and Slepian [S2], that among the $j$-simplices contained in a given ball, only the regular ones are largest.

In addition to the above standard formulas for simplex volumes, we need some other formulas that may not be quite so widely known. The following formula expresses the volume of a simplex in terms of its edge-lengths.

Theorem 1.3. Suppose that $S$ is a j-simplex in $\mathbb{R}^{n}$ with vertices $v_{1}, \ldots, v_{j+1}$, Let $B=\left(\beta_{i k}\right)$ denote the $(j+1) \times(j+1)$ matrix given by $\beta_{i k}=\left\|v_{i}-v_{k}\right\|^{2}$. Then

$$
2^{j}(j !)^{2} \operatorname{vol}^{2}(S)=|\operatorname{det}(\hat{B})|,
$$

where $\hat{B}$ is the $(j+2) \times(j+2)$ matrix obtained from $B$ by bordering $B$ with a top row $(0,1, \ldots, 1)$ and a left column $(0,1, \ldots, 1)^{T}$.

The determinant in Theorem 1.3 has become known as the Cayley-Menger determinant. Proofs of Theorem 1.3 can be found on pp. 124-125 of [S3] and p. 98 of [B]. For most of our purposes, the following closely related formula is more useful. It expresses the volume in terms of the Gram matrix formed from the inner products of the vertices.

Theorem 1.4. Suppose that $S$ is a j-simplex in $\mathbb{R}^{n}$ with $0 \in \operatorname{aff}(S)$, and $A$ is the $(j+1) \times n$ matrix whose rows list the coordinates of the vertices of $S$. Then

$$
(j !)^{2} \operatorname{vol}^{2}(S)=\operatorname{det}\left(J+A A^{T}\right),
$$

where $J$ is the $(j+1) \times(j \times 1)$ matrix whose entries are all 1 . If the origin is a vertex of $S$, then

$$
(j !)^{2} \operatorname{vol}^{2}(S)=\operatorname{det}\left(A_{0} A_{0}^{T}\right),
$$

where $A_{0}$ is formed from $A$ by discarding $A$ 's zero row.

Proof. Suppose first that the origin is a vertex of $S$. If $j=n$, then $A_{0}$ is a square matrix, a standard formula tells us that $(n !) \operatorname{vol}(S)=\left|\operatorname{det}\left(A_{0}\right)\right|$, and the stated 
conclusion follows from the fact that $\operatorname{det}\left(A_{0} A_{0}^{T}\right)=\operatorname{det}^{2}\left(A_{0}\right)$. If $j<n$, then, since the entries of $A_{0} A_{0}^{T}$ are inner products and these are invariant under rotation about the origin, we may assume without loss of generality that all the vertices of $S$ lie in the subspace of $\mathbb{R}^{n}$ consisting of points whose last $n-j$ coordinates are all zero. Form $B_{0}$ from $A_{0}$ by dropping the last $n-j$ columns of $A_{0}$. Then $B_{0}$ is a square matrix and $B_{0} B_{0}^{T}=A_{0} A_{0}^{T}$. This reduces the problem of proving the formula involving $A_{0}$ to the just-treated case in which $S$ is full-dimensional.

Now suppose that the origin is not a vertex of $S$ but does belong to the affine hull of $S$. Let $B$ denote the $(j+1) \times(n+1)$ matrix formed from $A$ by appending a column of 1 's. Then $B$ 's rows are the vertices of a $j$-simplex $S^{\prime}$ in $\mathbb{R}^{n+1}$ that is congruent to $S$ and whose affine hull is at distance 1 from the origin. Let $T$ denote the $(j+1)$-simplex $\operatorname{conv}\left(S^{\prime} \cup\{0\}\right)$. Then $\operatorname{vol}(T)=1 \cdot \operatorname{vol}\left(S^{\prime}\right) /(j+1)$ by a standard formula, and $((j+1) !)^{2} \operatorname{vol}^{2}(T)=\operatorname{det}\left(B B^{T}\right)$ by the result of the preceding paragraph. Since $\operatorname{vol}(S)=\operatorname{vol}\left(S^{\prime}\right)$ and $B B^{T}=J+A A^{T}$, the desired conclusion follows.

For any two subsets $X$ and $Y$ of $\mathbb{R}^{n}$, we define the distance

$$
\operatorname{dist}(X, Y)=\inf \{|| x-y \|: x \in X, y \in Y\}
$$

When both sets are flats (affine subspaces), we say that they are skew-orthogonal provided that $\operatorname{dist}(X, Y)>0$, there is a unique pair of points $x_{0} \in X$, and $y_{0} \in Y$ such that $\left\|x_{0}-y_{0}\right\|=\operatorname{dist}(X, Y)$, and the subspaces $X-x_{0}$ and $Y-y_{0}$ are mutually orthogonal.

Lemma 1.5. If $L$ and $M$ are orthogonal linear subspaces of $\mathbb{R}^{n}, X$ and $Y$ are affine subspaces of $L$ and $M$, respectively, and $0 \notin X \cap Y$, then $X$ and $Y$ are skew-orthogonal with

$$
\operatorname{dist}^{2}(X, Y)=\operatorname{dist}^{2}(0, X)+\operatorname{dist}^{2}(0, Y) .
$$

Proof. Let $x_{0}$ and $y_{0}$ be the unique points of $X$ and $Y$, respectively, that are closest to the origin. Then $x_{0} \neq y_{0}$, and for each $x \in X$ and $y \in Y$ we have $x-x_{0} \in L$ and $y-y_{0} \in M$, so $\left\langle x-x_{0}, y-y_{0}\right\rangle=0$. Since $\langle x, y\rangle=0$, we have

$$
\|x-y\|^{2}=\|x\|^{2}+\|y\|^{2} \geq\left\|x_{0}\right\|^{2}+\left\|y_{0}\right\|^{2}
$$

with equality on the right if and only if $x=x_{0}$ and $y=y_{0}$. The stated conclusion follows.

Theorem 1.6. Suppose that $F$ is a j-simplex in $\mathbb{R}^{n}, G$ is a $k$-simplex in $\mathbb{R}^{n}$, and the flats $\operatorname{aff}(F)$ and $\operatorname{aff}(G)$ are skew-orthogonal. Then the convex hull $\operatorname{conv}(F \cup G)$ is a 
$(j+k+1)$-simplex whose volume is equal to

$$
\frac{j ! k !}{(j+k+1) !} \operatorname{dist}(\operatorname{aff}(F), \operatorname{aff}(G)) \operatorname{vol}(F) \operatorname{vol}(G)
$$

Proof. With $X=\operatorname{aff}(F)$ and $Y=\operatorname{aff}(G)$, let the points $x_{0}$ and $y_{0}$ be those associated with the definition of skew-orthogonality and let $\delta=\left\|x_{0}-y_{0}\right\|$. Since the hypotheses are invariant under rigid motions, we may assume without loss of generality that $\mathbb{R}^{n}=\mathbb{R}^{1} \times \mathbb{R}^{j} \times \mathbb{R}^{k}$ with $x_{0}=\{0\} \times\{0\}^{j} \times\{0\}^{k}$ (the origin), $y_{0}=$ $\{\delta\} \times\{0\}^{j} \times\{0\}^{k}, X=\{0\} \times \mathbb{R}^{j} \times\{0\}^{k}$, and $Y=\{\delta\} \times\{0\}^{j} \times \mathbb{R}^{k}$. Let $\left\{v_{1}, \ldots, v_{j+1}\right\}$ and $\left\{w_{1}, \ldots, w_{k+1}\right\}$ be the vertex sets of $F$ and $G$ taken in $\mathbb{R}^{j}$ and $\mathbb{R}^{k}$, respectively. Then

$$
(j+k+1) ! \operatorname{vol}(\operatorname{conv}(F \cup G))=|\operatorname{det}(M)|,
$$

where

$$
M=\left(\begin{array}{cccc}
1 & 0 & v_{1}^{T} & 0 \\
\vdots & \vdots & \vdots & \vdots \\
1 & 0 & v_{j+1}^{T} & 0 \\
1 & \delta & 0 & w_{1}^{T} \\
\vdots & \vdots & \vdots & \vdots \\
1 & \delta & 0 & w_{k+1}^{T}
\end{array}\right)
$$

With the aid of row operations, we see that

$$
\begin{aligned}
\operatorname{det}(M) & =\operatorname{det}\left(\begin{array}{cccc}
1 & 0 & v_{1}^{T} & 0 \\
0 & 0 & v_{2}^{T}-v_{1}^{T} & 0 \\
\vdots & \vdots & \vdots & \vdots \\
0 & 0 & v_{j+1}^{T}-v_{1}^{T} & 0 \\
0 & \delta & -v_{1}^{T} & w_{1}^{T} \\
\vdots & \vdots & \vdots & \vdots \\
0 & \delta & -v_{1}^{T} & w_{k+1}^{T}
\end{array}\right)=\operatorname{det}\left(\begin{array}{ccc}
0 & v_{2}^{T}-v_{1}^{T} & 0 \\
\vdots & \vdots & \vdots \\
0 & v_{j+1}^{T}-v_{1}^{T} & 0 \\
\delta & -v_{1}^{T} & w_{1}^{T} \\
0 & 0 & w_{2}^{T}-w_{1}^{T} \\
\vdots & \vdots & \vdots \\
0 & 0 & w_{k+1}^{T}-w_{1}^{T}
\end{array}\right) \\
& =(-1)^{j} \delta \operatorname{det}\left(\begin{array}{c}
v_{2}^{T}-v_{1}^{T} \\
\vdots \\
v_{j+1}^{T}-v_{1}^{T}
\end{array}\right) \operatorname{det}\left(\begin{array}{c}
w_{2}^{T}-w_{1}^{T} \\
\vdots \\
w_{k+1}^{T}-w_{1}^{T}
\end{array}\right) \\
& =(-1)^{j} j ! \delta \operatorname{vol}(S) k ! \operatorname{vol}(T) .
\end{aligned}
$$




\section{Largest and Stable Simplices in Convex Bodies}

Suppose that $S$ is a $j$-simplex in a body $C$, and $W$ is a (possibly empty) subset of the set of vertices of $S$. Then we say that $S$ is $W$-largest in $C$ if $\operatorname{vol}(S) \geq \operatorname{vol}\left(S^{\prime}\right)$ for each $j$-simplex $S^{\prime}$ in $C$ whose vertex set contains $W$. If $\operatorname{vol}(S) \geq \operatorname{vol}\left(S^{\prime}\right)(\operatorname{resp} . \operatorname{vol}(S)>$ $\operatorname{vol}\left(S^{\prime}\right)$ ) whenever $S^{\prime}$ is a $j$-simplex in $C$ such that each point of $W$ is a vertex of $S^{\prime}$ and $S^{\prime}$ is obtained from $S$ by moving a single vertex to a new position in $C$, then we say that $S$ is $W$-stable (resp. $W$-rigid) in $C$; and $S$ is $W$-bound to $C$ if each vertex of $S$ that is not in $W$ is an extreme point of $C$. (When $W$ is a singleton $\{w\}$, we write $w$-largest, $w$-stable, etc.)

Note that when the set $W$ is empty, the above notions become the notions of largest, stable, rigid, and bound defined earlier. Note also that $W$-rigid implies $W$-stable, and $W$-largest implies $W$-stable. However, easy two-dimensional examples show that in general rigid does not imply largest and largest does not imply rigid (see Theorem 3.4 and the examples following its proof). Also, it is shown in [HKL] that for bound $n$-simplices in an $n$-cube, rigid implies largest if and only if $n \leq 4$, largest implies rigid if $n \in\{3,4,5\}$ and also if an $(n+1) \times(n+1)$ Hadamard matrix exists, and largest does not imply rigid when $n \in\{2,6,10\}$. (It is conjectured in [HKL] that, for each $n \equiv 2(\bmod 4)$, the $n$-cube contains bound largest $n$-simplices that are not rigid.)

As the term is used here, a face of a convex set $C$ is a set that is either empty or is the union, for some point $p \in C$, of all segments in $C$ that have $p$ as an inner point. The extreme points of $C$ are just the faces of dimension zero. When a body $C$ is a polytope, its faces (other than $\varnothing$ and $C$ itself) are precisely the sets that are formed by intersecting $C$ with one of its supporting hyperplanes.

Lemma 2.1. Suppose that $S$ is a $W$-stable j-simplex in a body $C$, and $v$ is a vertex of $S$ that does not belong to $W$. If $v$ is an extreme point of the intersection $\operatorname{aff}(S) \cap C$, then $v$ is an extreme point of $C$. More generally, if $v$ lies in the relative interior of a face $G$ of the set $\operatorname{aff}(S) \cap C$, then $G$ is a face of $C$.

Proof. Let $F$ denote the facet of $S$ that misses $v$, let $A$ denote the affine hull aff $(F)$, and for each point $x$ of the containing space let

$$
\varphi(x)=\min \{\|x-a\|: a \in A\} .
$$

Suppose that $v$ lies in the relative interior of a face $G$ of $\operatorname{aff}(S) \cap C$, but $G$ is not a face of $C$. Then $v$ is an inner point of a segment $[p, q]$ that is contained in $C$ but intersects aff $(S)$ only at $v$. It is easy to see that $[p, q]$ is not parallel to $A$, so the restriction of $\varphi$ to $[p, q]$ is strictly convex and it follows that $p$ or $q$ is farther from $A$ than $v$ is. Replacing $v$ by this farther point produces the vertex set of a $j$-simplex $S^{\prime}$ in $C$ that has larger $j$-measure than $S$. Since $W$ is contained in the vertex set of $S^{\prime}$, this contradicts the assumed $W$-stability of $S$.

Theorem 2.2. If $S$ is a W-largest $j$-simplex in a body $C$, then the intersection 
$C \cap \operatorname{aff}(S)$ contains a largest $j$-simplex that is $W$-bound. If $C$ contains more than one $W$-largest $j$-simplex, then more than one such $j$-simplex is $W$-bound.

Proof. In view of Lemma 2.1, it suffices for both assertions of the theorem to consider the case in which $j=n=\operatorname{dim}(C)$.

Let $S$ be an arbitrary $W$-largest $j$-simplex in $S$, let $v$ be an arbitrary vertex of $S$ that does not belong to $W$, and let $F$ and $A$ be as in the preceding proof. Let $H$ denote the translate of the hyperplane $A$ that supports $C$ and is on the same side of $A$ as $v$ is. The intersection $H \cap C$ includes an extreme point of $C$, and replacing $v$ by such an extreme point yields another largest simplex. A suitable repetition of this replacement process leads to the first conclusion.

Now suppose that $T$ is a $W$-largest $W$-bound $j$-simplex in $C$ that is different from $S$. Since $S$ and $T$ are both largest, $S$ is not contained in $T$ and hence there is a vertex $v$ of $S$ that does not belong to $T$. If $v$ is an extreme point of $C$, let $v^{\prime}=v$. If $v$ is not an extreme point of $C$, note that the intersection $H \cap C$ of the preceding paragraph must contain an extreme point $v^{\prime}$ of $C$ that does not belong to $T$. Now let $S^{\prime}$ denote the simplex obtained from $S$ by replacing $v$ with $v^{\prime}$, and apply the process of the preceding paragraph to the remaining vertices of $S^{\prime}$ that do not belong to $W$ and are not extreme points of $C$. The result is a $W$-largest $W$-bound $j$-simplex that is different from $T$.

Theorem 2.3. Suppose that $w$ is a point of a body $C$, and $S$ is a $w$-stable j-simplex in $C$. Then at least one vertex of $S$ other than $w$ is an extreme point of $C$.

Proof. We may again assume that $j=n=\operatorname{dim}(C)$, and assume also that $w$ is the origin. Then $(n !) \operatorname{vol}(S)=\left|\operatorname{det}\left(A_{0}\right)\right|$, where $A_{0}$ is the $n \times n$ matrix whose rows list the coordinates of the remaining vertices $v_{1}, \ldots, v_{n}$ of $S$. If none of these vertices is an extreme point of $C$, then for $1 \leq i \leq n$ there is a nonzero vector $b_{i}$ such that the segment $\left[v_{i}-b_{i}, v_{i}+b_{i}\right]$ is contained in $C$. Since $S$ is $w$-stable, this segment must be parallel to the facet of $S$ that omits $v_{i}$. From that fact, as applied successively to the various choices of $i$, it follows that the volume of the simplex with vertex set $\left\{0, v_{1}+\tau_{1} b_{1}, \ldots, v_{n}+\tau_{n} b_{n}\right\}$ is independent of the choice of $\tau_{1}, \ldots, \tau_{n}$. However, then, with $B^{T}=\left(b_{1}, \ldots, b_{n}\right)$ and using the determinantal formula for the volume of an $n$-simplex in $\mathbb{R}^{n}$ having a vertex at the origin, we conclude that the value of the determinant

$$
\operatorname{det}\left(A_{0}+\operatorname{diag}\left(\tau_{1}, \ldots, \tau_{n}\right) B\right)
$$

is independent of the $\tau_{i}$. It then follows from Theorem 1.1 that one of the $b_{i}$ is zero, and this contradiction completes the proof.

Corollary 2.4. If $S$ is a stable $j$-simplex in a body $C$, then at least two vertices of $S$ are extreme points of $C$.

Proof. By Theorem 2.3, at least one vertex (call it $w$ ) of $S$ is an extreme point of $C$. If the simplex $S$ is stable, then of course it is $w$-stable, and hence (again by Theorem 2.3) there is another vertex of $S$ that is an extreme point of $C$. 
The number "two" in Corollary 2.4 cannot in general be increased, even when the containing $n$-body $C$ is a high-dimensional polytope with many vertices and $S$ is a largest $n$-simplex in $C$. That is shown by a construction at the end of this section. However, the following result is often useful in restricting the locations of the vertices of stable simplices in a given body.

Theorem 2.5. Let $v_{0}, \ldots, v_{j}$ denote the vertices of a $W$-stable $j$-simplex $S$ in a given body $C$. With $r<j$, suppose that $W \subset\left\{v_{0}, \ldots, v_{r}\right\}$, that each of the points $v_{0}, \ldots, v_{r}$ which does not belong to $W$ is an extreme point of $C$, and that none of the points $v_{r+1}, \ldots, v_{j}$ is an extreme point of $C$. Then, for some $i$ with $r<i \leq j$, it is true that each segment in $C$ that crosses $v_{i}$ is parallel to the affine hull of $\left\{v_{0}, \ldots, v_{r}\right\}$.

Proof. Taking $v_{0}=0$, and using the same determinantal volume formula as in the proof of Theorem 2.3, we see that Theorem 2.5 follows immediately from Theorem 1.2.

The next three results concern stable, rigid, and largest simplices in "typical" polytopes and convex bodies. For each integer $n \geq 2$, let $\mathscr{C}^{n}$ denote the space of all bodies in $\mathbb{R}^{n}$, metrized by the Hausdorff metric. For each integer $m>n$, let $\mathscr{P}_{v}^{n}(m)$ (resp. $\mathscr{P}_{f}^{n}(m)$ ) denote the collection of all polytopes $P \in \mathscr{C}^{n}$ such that $P$ has at most $m$ vertices (resp. at most $m$ facets). Then $\mathscr{P}_{v}^{n}(m)$ and $\mathscr{P}_{f}^{n}(m)$ are both closed subsets of $\mathscr{C}^{n}$. The space $\mathscr{C}^{n}$ is not complete, but it is a dense $G_{\delta}$ subset of the space of all nonempty compact convex subsets of $\mathbb{R}^{n}$. Hence it follows from Baire's theorem that in each of $\mathscr{C}^{n}, \mathscr{P}_{v}^{n}(m)$, and $\mathscr{P}_{f}^{n}(m)$, the intersection of any sequence of dense $G_{\delta}$ subsets is itself a dense $G_{\delta}$ subset.

Theorem 2.6. In each of $\mathscr{C}^{n}$ and $\mathscr{P}_{v}^{n}(m)$, a dense $G_{\delta}$ subset is formed by the set of all members in which, for $1 \leq j \leq n$, there is a unique largest $j$-simplex.

Proof. In view of Theorem 2.2, attention may be restricted to bound simplices. We deal first with $\mathscr{E}^{n}$. For each choice of positive integers $j$ and $h$ with $1 \leq j \leq n$, let $\mathscr{C}^{n}(j, h)$ denote the collection of all $C \in \mathscr{E}^{n}$ such that $C$ contains two bound $j$-simplices $S$ and $T$ that satisfy the following three conditions:

$$
\operatorname{vol}(S)=\operatorname{vol}(T) \text {. }
$$

The Hausdorff distance between $S$ and $T$ is at least $1 / h$.

The distance between any two vertices of $S$ is at least $1 / h$ and the distance between any two vertices of $T$ is at least $1 / h$.

A routine argument shows that, for each $j$ and $h$, the collection $\mathscr{E}^{n}(j, h)$ is a closed subset of $\mathscr{C}^{n}$. The complement

$$
\mathscr{K}=\mathscr{E}^{n} \backslash \bigcup_{j=1}^{n} \bigcup_{h=1}^{\infty} \mathscr{E}^{n}(j, h)
$$


is precisely the set of all members of $\mathscr{C}^{n}$ in which, for each $j$, there is a unique largest $j$-simplex. Hence it remains only to show that $\mathscr{K}$ is dense in $\mathscr{C}^{n}$.

Let $\mathscr{C}_{\mathrm{sc}}^{n}$ denote the set of all strictly convex members of $\mathscr{C}^{n}$, whence (by an observation of Klee [K4] and Gruber [G1]) $\mathscr{C}_{\mathrm{sc}}^{n}$ is a dense $G_{\delta}$ subset of $\mathscr{C}^{n}$. In view of Baire's theorem, to establish $\mathscr{K}$ s density in $\mathscr{C}^{n}$ it suffices to prove for each fixed $j$ that each member $C$ of $\mathscr{E}_{\mathrm{sc}}^{n}$ can be closely approximated by a member of $\mathscr{K}$.

Let $S$ be a largest $j$-simplex in $C$. Since $C$ is strictly convex, the centroid of $S$ is interior to $C$ and may without loss of generality be assumed to be the origin. Let $L$ denote the $j$-dimensional linear hull of $S$, let $M$ denote a complementary linear $(n-j)$-dimensional subspace of $\mathbb{R}^{n}$, and let $T$ denote an $(n-j)$-simplex such that, relative to $M$,

$$
0 \in \operatorname{int}(T) \text { and } T \subset \operatorname{int}\left(C \cap M^{\prime}\right)
$$

Let $K=\operatorname{conv}(S \cup T)$, let $\kappa$ denote the gauge-functional of $K$, and let $\gamma$ denote the gauge-functional of $C$. For $0<\lambda<1$, let

$$
\mu_{\lambda}=(1-\lambda) \gamma+\lambda \kappa \text { and } C_{\lambda}=\left\{x \in \mathbb{R}^{n}: \mu_{\lambda}(x) \leq 1\right\}
$$

Then $S \subset C_{\lambda} \subset C$, and $C_{\lambda} \rightarrow C$ as $\lambda \rightarrow 0$. Note also that if $R$ is any ray that issues from the origin and passes through a point of $\operatorname{bd}(C)$ that does not belong to $\operatorname{bd}(S)$, then $R$ hits $\mathrm{bd}\left(C_{\lambda}\right)$ before it hits $\operatorname{bd}(C)$.

It is obvious that $S$ is a largest $j$-simplex in $C_{\lambda}$, and that any other largest $j$-simplex $S^{\prime}$ in $C_{\lambda}$ has at least one vertex $v$ that does not belong to $S$. However, then $\eta v \in C$ for some $\eta>1$, and from this (since $0 \in \operatorname{int}(C)$ ) it follows that $v \in \operatorname{int}(C$ ). Hence $v$ can be moved to a point of $C$ that is farther than $v$ is from the complementary facet of $S^{\prime}$, thereby producing in $C$ a $j$-simplex of greater volume than $S^{\prime}$. This is a contradiction, and it completes the proof for the case of $\mathscr{E}^{n}$.

The argument for $\mathscr{P}_{v}^{n}(m)$ is similar but simpler. Suppose that $P \in \mathscr{P}_{v}^{n}(m)$ with $0 \in \operatorname{int}(P)$, and let $S$ be a bound largest $j$-simplex in $P$. Let $v_{0}, \ldots, v_{j}$ be the vertices of $S$, and let $v_{j+1}, \ldots, v_{m-1}$ be the remaining vertices of $P$. For $0 \leq \eta<1$, let

$$
P_{\eta}=\operatorname{conv}\left\{v_{0}, \ldots, v_{j}, \eta v_{j+1}, \ldots, \eta v_{m-1}\right\}
$$

Then $S \subset P_{\eta} \subset P, P_{\eta} \rightarrow P$ as $\eta \rightarrow 1$, and an argument like the one above shows that $S$ is the unique largest $j$-simplex in each $P_{\eta}$.

For $\mathscr{P}_{v}^{n}(m)$, a considerably stronger theorem can be proved. For each finite subset $X$ of $\mathbb{R}^{n}$, let $\mathscr{A}_{X}$ denote the collection of all affinely independent sets consisting of two or more points of $X$, and let

$$
\mathscr{M}_{X}=\left\{\operatorname{vol}(\operatorname{conv}(A)): A \subset \mathscr{A}_{X}\right\}
$$

The set $X$ is called generic if $\left|\mathscr{A}_{X}\right|=\left|\mathscr{M}_{X}\right|$-in other words, no two simplices with vertices in $X$ have the same volume (even when they differ in dimension). A polytope $P$ is called generic if its vertex set is generic. 
Theorem 2.7. If $P$ is generic, then each stable simplex (of whatever dimension) in $P$ is bound and rigid, and for each $j$ with $1 \leq j \leq \operatorname{dim}(P)$ there is a unique largest $j$-simplex in $P$.

Proof. Once we have proved that each stable simplex is bound, the other assertions will follow directly from the assumption that $P$ is generic. Suppose, then, that $v_{0}, \ldots, v_{j}$ are the vertices of a stable $j$-simplex $S$ in $P$, where each of the points $v_{0}, \ldots, v_{r}$ is a vertex of $\boldsymbol{P}$ but none of the points $v_{r+1}, \ldots, v_{j}$ is a vertex of $\boldsymbol{P}$. Then $r \geq 1$ by Corollary 2.4 , and we want to show that $r=j$.

Suppose that $r<j$. Then by Theorem 2.5 there is an index $i$ with $r<i \leq j$ such that each segment in $P$ that crosses $v_{i}$ is parallel to the flat $H=\operatorname{aff}\left(\left\{v_{0}, \ldots, v_{r}\right\}\right)$. Let $F$ denote the smallest face of $P$ that contains the point $v_{i}$. Then the affine hull $\operatorname{aff}(F)$ is at least one-dimensional, and since each line in $\operatorname{aff}(F)$ is parallel to a segment in $P$ that crosses $v_{i}$, it follows that each line $L$ in aff $(F)$ admits a translate that is contained in $H$. However, some such $L$ contains two vertices $u$ and $w$ of $F$, and these are also vertices of $P$. Since the two points $u$ and $w$ are at the same distance from the flat $H$, the two $(r+1)$-simplices

$$
\operatorname{conv}\left\{u, v_{0}, \ldots, v_{r}\right\} \text { and } \operatorname{conv}\left\{w, v_{0}, \ldots, v_{r}\right\}
$$

are of the same $(r+1)$-measure. This contradicts the hypothesis that $\boldsymbol{P}$ is generic.

The following result justifies our use of the term "generic."

Theorem 2.8. The generic members of $\mathscr{P}_{v}^{n}(m)$ form a dense $G_{\delta}$ subset of $\mathscr{P}_{v}^{n}(m)$.

Proof. Let $\mathscr{Q}$ denote the set of all members of $\mathscr{P}_{v}^{n}(m)$ that have precisely $m$ vertices. By a routine argument involving the distance of a vertex from the convex hull of the remaining vertices, $\mathscr{Q}$ is a dense $G_{\delta}$ subset of $\mathscr{P}_{v}^{n}(\mathrm{~m})$. It suffices, therefore, to deal with the subcollection $\mathscr{Q}$. This has the advantage that a sequence $Q_{1}, Q_{2}, \ldots$ of members of $\mathscr{Q}$ converges to a limit $Q_{0} \in \mathscr{Q}$ if and only if the vertex sets of the $Q_{i}$ converge (in the Hausdorff metric) to $Q_{0}$ 's vertex set.

Consider an arbitrary member $Q_{0}$ and $\mathscr{Q}$ and an arbitrary $\varepsilon>0$. As is well known (see [G2]), there is an $m$-vertex polytope $Q$ such that the Hausdorff distance between $Q$ and $Q_{0}$ is less than $\varepsilon / 2$ and $Q$ 's vertex set $\left\{v_{1}, \ldots, v_{m}\right\}$ is in general position (i.e., no hyperplane contains more than $n$ of the $v_{i}$ ). Further, there is $\tau \in] 0, \varepsilon / 2[$ for which the following is true:

$$
\operatorname{conv}(W) \in \mathscr{Q} \quad \text { for each set } \quad W=\left\{w_{1}, \ldots, w_{m}\right\}
$$

such that $\left\|w_{i}-v_{i}\right\|<\tau$ for all $i$.

We now perturb each point $v_{i}$ by less than $\eta$ so as to produce a set $W$ that is generic. Since the resulting polytope is still within $\varepsilon$ of $Q_{0}$, that will yield the desired conclusion. 
Let $w_{1}=v_{1}$ and $w_{2}=v_{2}$. For $3 \leq i \leq m$, proceed as follows after having produced the generic set $\left\{w_{1}, \ldots, w_{i-1}\right\}$. For each finite set $Y \in \mathscr{A}_{\left(w_{1}, \ldots, w_{i-1}\right)}$ of cardinality $|Y| \leq n$, and each $\eta \in \mathscr{M}_{\left\{w_{1}, \ldots, w_{t-1}\right\}}$, let

$$
Z(Y, \eta)=\left\{p \in \mathbb{R}^{n}: \eta|Y|=\operatorname{dist}(p, \operatorname{aff}(Y)) \operatorname{vol}(\operatorname{conv}(Y))\right\}
$$

Then the set $Z(Y, \eta)$ consists of all points $p \in \mathbb{R}^{n}$ such that the $|Y|$-simplex with vertex set $Y \cup\{p\}$ has volume $\eta$. When $|Y|<n$, this set is an $(n-1)$-manifold (more precisely, a cylinder) in $\mathbb{R}^{n}$, and when $|Y|=n$ it is the union of two parallel hyperplanes. In each case, $Z(Y, \eta)$ is a closed nowhere-dense subset of $\mathbb{R}^{n}$, and hence (since there are only finitely many possibilities for the pair $(Y, \eta)$ ) the same is true of the union $U_{(Y, \eta)} Z(Y, \eta)$. Thus the set

$$
\left\{w \in \mathbb{R}^{n}:\left\|w-v_{i}\right\|<\tau\right\} \backslash \underset{\substack{Y \in \mathscr{A}_{\left\{w_{1}, \ldots, w_{i-1},\right.},|Y| \leq n \\ \eta \in \mathscr{M}_{\left(w_{1}, \ldots, w_{i}, 1\right\}}}}{\bigcup} Z(Y, \eta)
$$

is nonempty, and choosing $w_{i}$ from this set results in the generic set $\left\{w_{1}, \ldots, w_{i}\right\}$. Hence the process can be continued to produce the desired set $W$.

It seems clear that a result similar to Theorem 2.8 holds for $\mathscr{P}_{f}^{n}(m)$, but as the formal argument for this appears to be quite ponderous, we do not include it.

To end this section we show how to construct an $n$-body $C_{\varepsilon}$ that contains largest $n$-simplices for which only two vertices are extreme points of $C_{\varepsilon}$. For an arbitrary $(n-1)$-body $B$ (and a sufficiently small $\varepsilon$ ), $C_{\varepsilon}$ consists of a cylinder of height $2 \varepsilon$ over $B$, capped on each end by a cone of height $1-\varepsilon$. Note that when $B$ is a polytope, so is $C_{\varepsilon}$.

Example 2.9. With $n \geq 2$, suppose that $H$ is a hyperplane through the origin in Euclidean $n$-space, $u$ is a unit vector orthogonal to $H$, and $B$ is an ( $n-1)$-body in $H$ that contains the origin. Let $\beta$ denote the volume $((n-1)$-measure $)$ of $B, \sigma$ the volume of a largest $(n-1)$-simplex in $B$, and $\sigma_{0}$ the volume of a 0 -largest $(n-1)$-simplex in $B$. Our construction requires that $\sigma / \sigma_{0}<2$, a condition that is certainly satisfied if the point 0 is sufficiently close to a vertex of a largest simplex in $B$. (See Section 7 for an open problem concerning the range of $\sigma_{0} / \sigma$ when 0 is $B$ 's center of symmetry.)

For $0<\varepsilon<1$, let

$$
B_{\varepsilon}=B+[-\varepsilon u, \varepsilon u] \text { and } C_{\varepsilon}=\operatorname{conv}\left(B_{\varepsilon} \cup[-u, u]\right) \text {. }
$$

Then $B_{\varepsilon}$ is a cylinder of volume ( $n$-measure) $2 \varepsilon \beta$ and $C_{\varepsilon}$ is the result of adding a conical cap of height $1-\varepsilon$ over each end of $B_{\varepsilon}$. We prove that if $2 \sigma_{0}>\sigma$, then, for all sufficiently small $\varepsilon>0, C_{\varepsilon}$ contains a largest $n$-simplex whose vertices other than $-u$ and $u$ all belong to the set $B$ and hence are not extreme points of $C_{\varepsilon}$. 
Let $V$ denote the vertex set of a bound largest $n$-simplex $T$ in $C_{E}$. We claim the following:

(i) If $V$ omits both $-u$ and $u$, then $\operatorname{vol}(T)<\beta \varepsilon$.

(ii) If $V$ includes only one of $-u$ and $u$, then

$$
\operatorname{vol}(T) \leq\left(\frac{1+\varepsilon}{1-\varepsilon}\right)^{d} \frac{\sigma}{n} .
$$

(iii) If $V$ includes both $-u$ and $u$, then $\operatorname{vol}(T) \geq 2 \sigma_{0} / n$.

Assertion (i) is obvious, for then $T \subset B_{\varepsilon}$. To justify (iii), let $S$ be a 0-largest $(n-1)$-simplex in $B$ and note that the set conv $(S \cup[-u, u])$ is an $n$-simplex in $C_{\varepsilon}$. To justify (ii), assume without loss of generality that

$$
u \in T \subset \operatorname{conv}\left(B_{\varepsilon} \cup\{u\}\right)
$$

and note that the set $B+\varepsilon u$ is intersected by each of the $n$ rays that issue from $u$ along an edge of $T$. The $n$ points of intersection are the vertices of an $(n-1)$-simplex $S$ in $B+\varepsilon$, and of course the volume of $S$ is at most $\sigma$. The $n$ vertices of $T$ other than $u$ all lie in the intersections of these rays with the strip $H+[-\varepsilon u, \varepsilon u]$. Hence the simplex $T$ is contained in the result of dilating the $\operatorname{simplex} \operatorname{conv}(S \cup\{u\})$ by a factor of $(1+\varepsilon) /(1-\varepsilon)$ about its vertex $u$, and that yields the volume bound in assertion (ii).

Now note that as $\varepsilon \rightarrow 0$, the upper bounds in (i) and (ii) and the lower bound in (iii) converge respectively to 0 , to $\sigma / n$, and to $2 \sigma_{0} / n$. Hence if $2 \sigma_{0}>\sigma$, the third bound eventually dominates. It follows that, for all sufficiently small $\varepsilon>0$, each bound largest simplex in $C_{\varepsilon}$ contains the segment $[-u, u]$.

Now assume that $H$ is the canonically embedded $\mathbb{R}^{n-1}$ in $\mathbb{R}^{n}$ and that $u$ is the last of the standard basis vectors. Let $T$ be a bound largest $n$-simplex in $C_{\varepsilon}$, with vertices

$$
v_{0}=-u, \quad v_{n}=u, \text { and } v_{1}, \ldots, v_{n-1} \in B+\{-\varepsilon u, \varepsilon u\} .
$$

For $0<i<n$, let $v_{i}=\left(\nu_{i 1}, \ldots, \nu_{i, n-1}, \nu_{i n}\right)^{T}$. Then $\operatorname{vol}(T)=|\operatorname{det}(A)| / n !$, where

$$
\boldsymbol{A}=\left(\begin{array}{ccccc}
1 & 0 & \ldots & 0 & -1 \\
1 & \nu_{11} & \ldots & \nu_{1, n-1} & \nu_{1 n} \\
\vdots & \vdots & \ddots & \vdots & \vdots \\
1 & \nu_{n-1,1} & \ldots & \nu_{n-1, n-1} & \nu_{n-1, n} \\
1 & 0 & \ldots & 0 & 1
\end{array}\right)
$$

Replacing each later row of $A$ by itself minus the first row, we see that

$$
\operatorname{det}(A)=2 \operatorname{det}\left(\begin{array}{ccc}
\nu_{11} & \cdots & \nu_{1, n-1} \\
\vdots & \ddots & \vdots \\
\nu_{n-1,1} & \cdots & \nu_{n-1, n-1}
\end{array}\right)
$$


In other words, the last coordinates of the vertices $v_{1}, \ldots, v_{n-1}$ are immaterial; only their projections onto $\mathbb{R}^{n-1}$ are relevant to $\operatorname{vol}(T)$. Hence we may replace all of these last coordinates by 0 's without changing the volume of the simplex. This shows that $2 \sigma_{0} / n$ is indeed the volume of the largest $n$-simplices in $C_{\varepsilon}$, and also that there is a largest $n$-simplex in $C_{\varepsilon}$ that contains only two extreme points of $C_{\varepsilon}$.

\section{Moving a Bound Simplex in a Convex Polytope}

It is assumed in this section that the containing body is an $n$-polytope $P$. When $S$ is a bound $j$-simplex in $P$, we define a move for $S$ as the operation of producing a new bound $j$-simplex $S^{\prime}$ by moving a single vertex $v$ of $S$ to a new position $v^{\prime}$. The move is fair if $\operatorname{vol}\left(S^{\prime}\right) \geq \operatorname{vol}(S)$ and $\operatorname{good}$ if $\operatorname{vol}\left(S^{\prime}\right)>\operatorname{vol}(S)$. For $1 \leq k \leq n$, the move is a $k$-move if $v^{\prime}$ lies on some $k$-face of $P$ that contains $v$. When the simplex $S$ is full-dimensional (i.e., when $\operatorname{dim}(S)=\operatorname{dim}(P)$ ), a move of $S$ is called an $R$-move if the vertices $v$ and $v^{\prime}$ are on opposite sides of the hyperplane that contains the remaining vertices of $S$. (Think of $R$ as standing for "reflection.")

For $1 \leq k \leq n$, a bound $j$-simplex $S$ in $P$ is $k$-stable (resp. $k$-rigid) if it does not admit a good (resp. fair) $k$-move. Thus $n$-stability and $n$-rigidity are precisely the stability and rigidity defined in the Introduction, while for $1 \leq k<n$ the $k$-stability and $k$-rigidity are less restrictive notions. The notions of $k$-stability and $k$-rigidity can be combined with the notions of $W$-stability and $W$-rigidity used in Section 2, leading to extensions of the results of the present section, but to keep matters simple we refrain from making that combination.

The following conditions on a polytope $P$ are satisfied by some polytopes and fail for others. They are all relevant to the attempt to find largest $j$-simplices in $P$ by starting with some $j$-simplex, attempting to improve it by successively moving one vertex at a time, then if necessary trying another starting $j$-simplex, etc.

$M_{1}(k, j)$ : If a bound $j$-simplex $S$ in $P$ is not largest, then $S$ admits a sequence of successive fair $k$-moves leading to a largest $j$-simplex.

$M_{2}(k, j)$ : If a bound $j$-simplex $S$ in $P$ admits a fair $k$-move, then it admits a sequence of successive fair $k$-moves ending in a good $k$-move.

$M_{3}(k, j)$ : If a bound $j$-simplex $S$ in $P$ is largest, it is $k$-rigid.

$B(j)$ : Each largest $j$-simplex in $P$ is bound.

Property $M_{1}(k, j)$ guarantees that, however far an initial bound $j$-simplex $S$ may be from maximizing the volume, a largest $j$-simplex can be reached from $S$ by following a sufficiently long sequence of fair $k$-moves. Hence there is no need (at least in theory) to try more than one $j$-simplex as the starting point of the search. Unfortunately, this seems to be a rare property in cases of interest. The existence of convex polygons lacking property $M_{1}(1,2)$ was relevant to the design of an algorithm by Dobkin and Snyder [DS] for finding a largest triangle in a given convex polygon. Each of the basic steps in their algorithm moves a single vertex of the maximumseeking triangle along an edge, but when $M_{1}(1,2)$ is lacking they are forced to employ moves that in our terminology are not fair. Also, it is proved in [HKL] 
that when the polytope in question is an $n$-cube, the properties $M_{1}(1, n), \ldots$, $M_{1}(n-1, n)$ are all present when $n \leq 4$ but all absent when $n \geq 5$.

An equivalent statement of property $M_{2}(k, j)$ is that each bound $j$-simplex in $P$ is either $k$-rigid or admits a sequence of successive fair $k$-moves leading to a $k$-rigid $j$-simplex. That is useful (when it occurs), for it means that the "dead end" $j$-simplices (those from which no further volume increase can be obtained by a sequence of fair $k$-moves) are precisely the ones that are $k$-rigid and hence can be recognized fairly easily by the use of local tests.

Property $M_{3}(k, j)$ makes it easier, when a largest bound $j$-simplex is reached, to recognize that this is the case. Even though $P$ always contains a largest $j$-simplex that is bound (Theorem 2.2), $P$ may also contain largest $j$-simplices that are not bound. Hence if we are seeking all largest $j$-simplices in $P$, it is important to know whether $P$ has the property $B(j)$.

Properties $M_{1}(k, j)-M_{3}(k, j)$ are naturally interpreted in terms of the nodelabeled digraph $\mathscr{M}(P, k, j)$ whose nodes are the bound $j$-simplices in $P$, with each node labeled by the volume of the corresponding simplex and with an arc $\left(S, S^{\prime}\right)$ directed from $S$ to $S^{\prime}$ if and only if $S^{\prime}$ is obtained from $S$ by a fair $k$-move. The $k$-rigid $j$-simplices are precisely the sinks in this digraph, so $M_{3}(k, j)$ asserts that all largest nodes are sinks. Property $M_{2}(k, j)$ says that each node of $\mathscr{M}(P, k, j)$ is a sink or is the start of a path that ends at a sink; this implies that all largest nodes are sinks (hence implies $M_{3}(k, j)$ ). Property $M_{1}(k, j)$ says that starting from any node, there is a path that leads to a largest node; this implies that each sink is a largest node.

Theorem 3.1. Suppose that $1 \leq k \leq n$ and $1 \leq j \leq n$. For each n-polytope $P$, property $M_{2}(k, j)$ implies property $M_{3}(k, j)$, and $M_{3}(k, j)$ implies $B(j)$.

Proof. The first implication is obvious, as was noted above. To establish the second implication, we show that if an $n$-polytope $P$ admits a largest $j$-simplex $S$ that is not bound, then there is a largest $j$-simplex $S^{\prime}$ that is bound and admits a fair 1-move. That is also a fair $k$-move for each $k$, thus contradicting property $M_{3}(k, j)$ and completing the proof.

Since largest $j$-simplices are stable, we see from Theorem 2.1 that it suffices to consider the case $j=n$. Now note that when stated in the language introduced in this section, the proof of Theorem 2.2 constructs from a largest $j$-simplex $S$ that is not bound a sequence of fair moves that ends with a largest bound $j$-simplex $S^{\prime}$. The final move replaces a vertex $v$ of the next-to-last simplex by a vertex $v^{\prime}$ of $P$ such that $v$ and $v^{\prime}$ lie together on a supporting hyperplane $H$ of $P$. Since all simplices of the sequence are largest, the entire face $F^{\prime}=H \cap P$ is parallel to the facet of $S^{\prime}$ that does not contain $v^{\prime}$. Hence every edge of $F^{\prime}$ can be used for a fair 1-move.

Now suppose that $F$ is a facet of a bound $j$-simplex $S$ in an $n$-polytope $P, v$ is the vertex of $S$ not on $F$, and $v^{\prime}$ is a different vertex of $P$. Then moving from $v$ to $v^{\prime}$ constitutes a fair move for $S$ if and only if

$$
\operatorname{dist}\left(v^{\prime}, \operatorname{aff}(F)\right) \geq \operatorname{dist}(v, \operatorname{aff}(F)),
$$


and a good move if and only if this inequality is strict. Hence the problem of finding good moves is closely connected to the problem of maximizing the convex quadratic function

$$
\varphi(x)=\operatorname{dist}^{2}(x, \operatorname{aff}(F))
$$

for $x$ constrained to lie in the polytope $P$. This connection is related to the practical difficulty, when $j<n$ and an $n$-polytope $P$ is presented as an intersection of half-spaces, of finding a largest $j$-simplex in $P$ or even recognizing when a given bound $j$-simplex is stable, or rigid, or largest. However, the next result and Theorem 4.3 show that the situation changes, at least for stability and rigidity, when $j=n$. Then the function $\xi=\varphi^{1 / 2}$ is the absolute value of an affine function and the methods of linear programming are available.

Proposition 3.2. Suppose that $v$ is a vertex of an $n$-simplex $S$ in an n-polytope $P$. If $S$ admits a fair or good move that involves $v$, then it admits such a 1-move or such an R-move.

Proof. Let $F$ denote the facet of $S$ that misses $v$, define $\xi(x)=\operatorname{dist}(x, \operatorname{aff}(F))$, and let $v^{\prime}$ denote the vertex of $P$ to which $v$ is taken by the assumed move. Suppose that the move is not an $R$-move. Then $v^{\prime}$ belongs to the half-space $Q$ that contains $v$ and is bounded by the hyperplane $\operatorname{aff}(F)$. As restricted to $Q$, the function $\xi$ is affine. With

$$
\mu=\max \{\xi(x): x \in P \cap Q\}
$$

note that $\xi(v) \leq \mu$ and let

$$
G=\{p \in P \cap Q: \xi(p)=\mu\}
$$

Then $G$ is a face of $P$. If $\xi(v)<\mu$ (which must be the case if the move from $v$ to $v^{\prime}$ was good), it follows from a basic theorem of linear programming that $P$ admits an edge-path from $v$ to $G$ along which $\xi$ is steadily increasing. Moving $v$ along the first edge of this path constitutes a good 1-move for $S$. In the remaining case, $v$ and $v^{\prime}$ both lie in $G, G$ contains an edge-path from $v$ to $v^{\prime}$, and moving $v$ along the first edge in this path constitutes a fair move for $S$.

The following result sets out an additional relationship between properties $B$ and $M_{3}$.

Proposition 3.3. The following is true for $n=2$ but false for $n \geq 3$ :

Whenever $P$ is an n-polytope such that each largest $n$-simplex in $P$ is bound, then each largest $n$-simplex in $P$ is rigid.

Proof. Let $P$ be an $n$-polytope in which each largest $n$-simplex is bound, and suppose that some largest $n$-simplex $S$ in $P$ fails to be rigid. Then a vertex $v$ of $S$ 
and a vertex $v^{\prime}$ of $P$ but not $S$ exist such that if $F$ is the facet of $S$ that misses $v$ and $S^{\prime}=\operatorname{conv}\left(F \cup\left\{v^{\prime}\right\}\right)$, then $\operatorname{vol}\left(S^{\prime}\right)=\operatorname{vol}(S)$. Since we are concerned only with volume ratios, and since these are invariant under nonsingular affine transformations, we may assume without loss of generality that $v=0$ and that the remaining vertices of $S$ are the standard basis vectors $e_{1}, \ldots, e_{n}$.

For each point $x=\left(\xi_{1}, \ldots, \xi_{n}\right)^{T} \in \mathbb{R}^{n}$, let

$$
\zeta(x)=\sum_{i=1}^{n} \xi_{i}
$$

and for each $\alpha \in \mathbb{R}$ let

$$
H_{\alpha}=\{x: \zeta(x)=\alpha\} \text {. }
$$

Then the vertex $v^{\prime}$ belongs to the hyperplane $H_{0}$ or to the hyperplane $H_{2}$. In the former case, the segment $\left[v, v^{\prime}\right]$ also belongs to $H_{0}$, and moving $v$ to the point $\frac{1}{2}\left(v+v^{\prime}\right)$ produces a largest $n$-simplex in $P$ that is not bound. Hence $v^{\prime} \in H_{2}$ and we have

$$
v^{\prime}=\left(\eta_{1}, \ldots, \eta_{n}\right)^{T} \quad \text { with } \quad \sum_{i=1}^{n} \eta_{i}=2
$$

For $1 \leq i \leq n$, let

$$
S_{i}=\operatorname{conv}\left(\left\{v, v^{\prime}\right\} \cup\left\{e_{k}: i \neq k\right\}\right) .
$$

Trivially, $\operatorname{vol}\left(S_{i}\right) / \operatorname{vol}(S)=\left|\eta_{i}\right|$.

Now suppose that $n \geq 3$, let $\eta_{i}=2 / n$ for each $i$, and set $P=S \cup S^{\prime}$. Then for each $i$ the volume of $S_{i}$ is less than that of $S$ and $S^{\prime}$, so $S$ and $S^{\prime}$ are both largest $n$-simplices in $P$ and being largest does not imply being rigid. However, it is not hard to verify that $S$ and $S^{\prime}$ are the only largest $n$-simplices in $P$, and hence all largest $n$-simplices are bound.

The case $n=2$ remains. There, from the facts that $S$ is largest, that $\operatorname{vol}\left(S_{i}\right) / \operatorname{vol}(S)=\left|\eta_{i}\right|$, and that $\eta_{1}+\eta_{2}=2$, it follows that $\eta_{1}=\eta_{2}=1$. Hence $P$ contains the unit square $Q_{2}=[0,1]^{2}$. If $P=Q_{2}$, a contradiction results from the fact that some largest triangles in $Q_{2}$ are not bound, and if $P \neq Q_{2}$ a contradiction results from the fact that $P$ then contains triangles larger than $S$.

We end this section with some additional examples, focusing on the case in which $k=1$ and $j=n=2$ (the case of triangles in convex polygons). Of the 16 possible combinations of truth-values for properties $M_{1}(1,2)-M_{3}(1,2)$ and $B(2), 11$ are excluded by Theorem 3.1, Proposition 3.3, and the obvious fact that when property $M_{1}(k, j)$ is present, $M_{2}(k, j)$ and $M_{3}(k, j)$ are equivalent. However, the remaining five possibilities do occur for suitably constructed polygons, as is shown by the following theorem and the examples that follow its proof. (Since the occurrence or non-occurrence of property $M_{3}(1,2)$ is a consequence of the occurrence or nonoccurrence of the other properties, it suffices to consider only $M_{1}(1,2), M_{2}(1,2)$, and $B(2)$.) 
Theorem 3.4. If $P_{m}$ is a regular $m$-gon in $\mathbb{R}^{2}$, then each bound triangle in $P_{m}$ that is not largest admits a sequence of successive good 1-moves leading to a largest triangle. Hence, $P_{m}$ has property $M_{1}(1,2)$ for all $m$. Now suppose that $v_{0}, v_{1}, \ldots, v_{m}\left(=v_{0}\right)$ are the vertices of $P_{m}$, arranged in an order of boundary traversal.

- If $m=3 k$, the largest triangles in $P_{m}$ are the triangle $\operatorname{conv}\left\{v_{0}, v_{k}, v_{2 k}\right\}$ and the other triangles in $P_{m}$ that are equivalent to this one under the rotations of $P_{m}$ about its centroid. Each largest triangle in $P_{m}$ is bound and rigid, and $P_{m}$ has each of the properties $M_{2}(1,2), M_{3}(1,2)$, and $B(2)$.

- If $m=3 k+r$ with $r \in\{1,2\}$, the largest triangles in $P_{m}$ are those of the form conv $\left\{v_{0}, v_{k-1+r}, w\right\}$ with $w \in\left[v_{2 k-1+r}, v_{2 k+r}\right]$ and the other triangles in $P_{m}$ that are equivalent to one of these under the rotations of $P_{m}$ about its centroid. No triangle in $P_{m}$ is rigid, and there are largest triangles that are not bound, so $P_{m}$ lacks each of the properties $M_{2}(1,2), M_{3}(1,2)$, and $B(2)$.

Proof. We begin by considering an arbitrary pair $u, v$ of vertices of $P_{m}$ and describing the largest bound triangles in $P_{m}$ that have the segment $[u, v]$ as a base. Let $u, x_{1}, \ldots, x_{t}, v$ be a list of the vertices of $P_{m}$ that appear in the given order of traversal from $u$ to $v$. Assume that $t \geq 1$ and set $h=\lfloor t / 2\rfloor+1$. For $1 \leq i \leq t$ let $\alpha_{i}$ denote the area of the triangle $\operatorname{conv}\left\{u, v, x_{i}\right\}$. Using the facts that $P_{m}$ is inscribed in a circle $C$, and that the perpendicular bisector of the segment $[u, v]$ is an axis of symmetry of both $P_{m}$ and $C$, it follows easily that one of the two following statements is correct:

- $t$ is odd; $\alpha_{i}$ is strictly monotone increasing for $i=1, \ldots, h$ and strictly monotone decreasing for $i=h, \ldots, t$.

- $t$ is even and $\alpha_{h}=\alpha_{h+1} ; \alpha_{i}$ is strictly monotone increasing for $i=1, \ldots, h$ and strictly monotone decreasing for $i=h+1, \ldots, t$.

Now consider any three vertices $u, v$, and $w$ of $P_{m}$, and let $T$ denote the triangle conv $\{u, v, w\}$. Let $a, b$, and $c$ denote the numbers of vertices of $P_{m}$ that appear (relative to the given order of traversal) respectively on the arc from $u$ to $v$, on the arc from $v$ to $w$, and on the arc from $w$ to $u$. Let $q=\max (|a-b|,|b-c|,|c-a|\}$. Using the observation in the preceding paragraph, it is easy to verify the following statements:

(i) The triangle $T$ admits a good 1-move if and only if $q \geq 2$.

(ii) If $q=1, T$ admits a fair 1 -move that is not good.

(iii) If $q=0, T$ is 1 -rigid.

Finally, consider an arbitrary bound triangle $T$ in $P_{m}$, let $(a, b, c)$ be the triple associated as indicated with $T$, and verify the following statements:

(iv) $a+b+c=m-3$.

(v) If $b>0$, then $T$ admits a 1-move resulting in a triangle whose triple is $(a+1, b-1, c)$ and also admits a 1-move resulting in a triangle whose triple is $(a, b-1, c+1)$.

It follows from (v) that for any bound triangle a sequence of good 1-moves exists 
leading to a triangle with triple $(a, b, c)$ for which $q \leq 1$. It then follows from (iv) that $q=0$ if and only if $m$ is a multiple of 3 . The proof can now be completed by a straightforward application of observations (i)-(iii).

We now complete the collection of examples promised before the statement of Theorem 3.4.

Examples 3.5. Let $T$ be a triangle whose centroid is the origin, and let $P=$ $\operatorname{conv}(T \cup-\lambda T)$ with $1<\lambda<2$. Then $P$ has properties $M_{2}(1,2), M_{3}(1,2)$, and $B(2)$ but not $M_{1}(1,2)$. (Also $T$ is a triangle in $P$ that is rigid but not largest.) Next, form $P^{\prime}$ by slightly truncating the previous $P$ at a vertex of the smaller triangle $T$. Then $P^{\prime}$ has properties $B(2)$ and $M_{3}(1,2)$ but neither $M_{1}(1,2)$ nor $M_{2}(1,2)$. Finally, form $P^{\prime \prime}$ by slightly truncating $P$ at a vertex of the larger triangle $\lambda T$ with a "cut" parallel to the opposite edge. Then properties $M_{1}(1,2)-M_{3}(1,2)$ and $B(2)$ all fail for $P^{\prime \prime}$.

\section{Computational Preliminaries, Tractability Results}

In Sections 4-6 we consider the computational complexity of various forms of the following problems that involve a function $\gamma: \mathbb{N} \rightarrow \mathbb{N}$ with $1 \leq \gamma(n) \leq n$ for each $n \in \mathbb{N}$ :

$\Pi_{1}$ : Given $n \in \mathbb{N}$ and an $n$-polytope $P \subset \mathbb{R}^{n}$, determine (the volume of) a largest $\gamma(n)$-simplex $S$ in $P$.

$\Pi_{2}$ : Given $n \in \mathbb{N}$ and an $n$-polytope $P \subset \mathbb{R}^{n}$, is the largest $\gamma(n)$-simplex in $P$ unique?

$\Pi_{3}$ : Given $n \in \mathbb{N}$, an $n$-polytope $P \subset \mathbb{R}^{n}$, and a bound $\gamma(n)$-simplex $S$ in $P$, is $S$ a largest $\gamma(n)$-simplex in $P$ ?

$\Pi_{4}$ : Given $n \in \mathbb{N}$, an $n$-polytope $P \subset \mathbb{R}^{n}$, and a bound $\gamma(n)$-simplex $S$ in $P$, is $S$ stable in $P$ ?

$\Pi_{5}$ : Given $n \in \mathbb{N}$, an $n$-polytope $P \subset \mathbb{R}^{n}$, and a bound $\gamma(n)$-simplex $S$ in $P$, is $S$ rigid in $P$ ?

In the special case $\gamma \equiv 1$, problem $\Pi_{1}$ asks for the diameter of $P$, and when $\gamma(n)=n$ we are interested in a largest full-dimensional simplex in $P$. Since the latter case is of fundamental importance here, we occasionally refer to it as MAXSimp.

Note that in each case, the dimension $n$ is part of the input. Thus we are concerned primarily with the case of variable dimension $n$ and in a sense with the asymptotic behavior of the problems as $n \rightarrow \infty$. References to a few results for fixed dimension $n$ are given at the end of this section.

We employ the standard binary or Turing machine model of computation (see [GJ]), in which the size of the input is defined as the length (number of bits) of the binary encoding needed to present the input data to a Turing machine, and the time-complexity of an algorithm is also defined in terms of the operation of a Turing machine. An $\mathscr{H}$-polytope is one that is given as an intersection of finitely many closed 
half-spaces, where each half-space is defined by means of a linear inequality in which all coefficients are rational numbers. A $\mathscr{V}$-polytope is one that is given as the convex hull of a finite set of points, where all the points have rational coordinates. It is assumed also that each of the relevant rational numbers is in its lowest terms, and that each presentation is irredundant. For more details on these matters, and for formulas for the sizes of rational presentations of polytopes, see, e.g., [GK3]. (Minor variations in the formulas for size do not affect our concerns here, which are with the contrast between polynomial-time computability and $\mathbb{N} P$-hardness.)

From a rational presentation of a polytope $P$ it is possible in polynomial time to produce an integral presentation of $P$ or of a dilated version of $P$, and from this it follows that for our purposes the distinction between integral and rational presentations is unimportant. That fact is used here without further comment.

Our complexity results are qualitative in the sense that they classify certain problems as being solvable in polynomial time, or (more often) they show that certain problems are $\mathbb{N} P$-hard. Since we focus on the case of variable dimension, it is necessary to distinguish between $\mathscr{V}$-polytopes and $\mathscr{H}$-polytopes. That is because for $\mathscr{H}$-presented $n$-polytopes with $m$ facets, the maximum possible number of vertices is

$$
\mu(m, n)=\left(\begin{array}{c}
m-\lfloor(n+1) / 2\rfloor \\
m-n
\end{array}\right)+\left(\begin{array}{c}
m-\lfloor(n+2) / 2\rfloor \\
m-n
\end{array}\right)
$$

(see [M3]), and this is also the maximum possible number of facets for a $\mathscr{V}$-presented $n$-polytope with $m$ vertices. When $n$ is fixed, the number of vertices is bounded by a polynomial in the number of facets, and vice versa, and it is possible to pass from either sort of presentation to the other in polynomial time. However, the degree of the polynomial goes to infinity with $n$. A consequence of this is that when the dimension $n$ is permitted to vary in a problem concerning polytopes, then the manner of presentation is often influential in determining whether the problem can be solved in polynomial time. For a variable dimension, even determining the number of vertices of a given $\mathscr{H}$-polytope-or the number of facets of a given $\mathscr{V}$-polytope-is a problem that is \#P-hard [L].

When $S$ is a $j$-simplex in $\mathbb{R}^{n}$ whose vertices have exclusively rational coordinates, it follows from Theorems 1.3 and 1.4 that the number $\operatorname{vol}^{2}(S)$ is rational; however, only when $j=n$ can we be sure (by a standard formula) that the number $\operatorname{vol}(S)$ is itself rational. This accounts for the fact that, in making specifications to fit the general problems $\Pi_{1}-\Pi_{5}$ easily into a decision-theoretic binary framework, we often work in terms of squares of volumes rather than the volumes themselves.

In this section we give the necessary specifications for dealing with the "easy" cases of problems $\Pi_{1}-\Pi_{5}$, and we state and prove the associated "tractability results." Sections 5 and 6 contain the specifications for proving that certain versions of $\Pi_{1}$ and $\Pi_{2}$ are $\mathbb{N P}$-complete or at least $\mathbb{N P}$-hard. (Also, see the conjecture in this section after the proof of Theorem 4.3.)

Note first that when $j$ is fixed but $n$ is variable, the number of bound $j$-simplices in a $\mathscr{V}$-polytope is bounded by a polynomial in $n$. This leads to the following simple results for problems $\Pi_{1}-\Pi_{5}$. 
Theorem 4.1. For each fixed $j$, the squares of the volumes of all bound $j$-simplices in a given n-dimensional $\mathscr{Y}$-polytope can be computed in polynomial time (even for varying $n$ ). Hence it can be decided in polynomial time exactly which of the bound $j$-simplices are stable, which are rigid, which are largest, and which volumes occur uniquely.

Proof. If $P$ has $m$ vertices, there are only $\left(\begin{array}{c}m \\ j+1\end{array}\right)$ sets of $j+1$ vertices for which the square of the volume ( $j$-measure) of the convex hull must be computed. This number is (for fixed $j$ ) polynomial in $m$, and each individual volume computation can be made in polynomial time using Theorem 1.3 or Theorem 1.4.

A similar result holds when $n-j$ is fixed and the excess of the number of vertices over $n$ is bounded.

Theorem 4.2. For each fuxed $j$ and $k$, the squares of the volumes of all bound $(n-j)$-simplices in a given $n$-dimensional $\mathscr{Y}$-polytope $P$ with at most $n+k$ vertices can be computed in polynomial time (even for varying $n$ ). Hence it can be decided in polynomial time exactly which of the bound $(n-j)$-simplices are stable, which are rigid, which are largest, and which volumes occur uniquely.

After the at most $\mu(m, n)$ vertices of an $n$-dimensional $\mathscr{H}$-polytope with $m$ facets have been found,

$$
\left(\begin{array}{c}
\mu(m, n) \\
j+1
\end{array}\right)=O\left(m^{\lfloor n / 2\rfloor(j+1)}\right) \leq O\left(m^{\lfloor n / 2\rfloor(n+1)}\right)
$$

volume computations suffice to carry out the tasks described in Theorems 4.1 and 4.2. This bound is not reassuring, but at least for each fixed $j$ and $n$ it is of polynomial growth as a function of $m$. The situation changes drastically when $P$ 's dimension $n$ is permitted to vary, and that is reflected in the hardness results of Section 6 . However, "polynomiality" does persist for the following variable-dimension decision problems of type $\Pi_{4}$ and $\Pi_{5}$ concerning full-dimensional simplices in $\mathscr{X}$-polytopes.

Theorem 4.3. There are polynomial-time algorithms for the following problems:

Instance: $\quad n \in \mathbb{N}$, an $n$-dimensional $\mathscr{X}$-polytope (or $\mathscr{Y}$-polytope) $P$ in $\mathbb{R}^{n}$, and a bound n-simplex $S$ in $P$.

Questions: Is $S$ stable in $P$ ? Is $S$ rigid in $P$ ?

Proof. For each vertex $v$ of $S$, let $\varphi_{v}$ denote the affine functional on $\mathbb{R}^{n}$ that has $\varphi_{v}(v)=1$ and vanishes on the facet of $S$ that misses $v$. Using a polynomial-time algorithm for linear programming [K3], [K1] when $P$ is an $\mathscr{X}$-polytope, and using direct evaluation at all vertices when $P$ is a $\mathscr{V}$-polytope, compute the minimum $\alpha_{v}$ and the maximum $\beta_{v}$ of $\varphi_{v}$ on $P$. Then $S$ is stable in $P$ if and only if, for all $v$, $\alpha_{v}=1$ and $\beta_{v} \geq-1$; and $S$ is rigid if and only if for each $v$ it is true that $\beta_{v}>-1$ and also that $v$ is the unique vertex of $P$ for which $\alpha_{v}=1$. This uniqueness can also be tested in polynomial time. 
We conjecture that unless $\mathbb{P}=\mathbb{N} \mathbb{P}$, Theorem 4.3 gives a full description of the situations in which, for a general bound $j$-simplex $S$ in a general $n$-dimensonal $\mathscr{Z}$-polytope $P$, it can be decided in polynomial time whether $S$ is stable in $P$, or rigid in $P$, or largest in $P$. It would not surprise us if, except for the situation covered by Theorem 4.3, these problems turn out to be $\mathbb{N} P$-hard even for $\mathscr{H}$-polytopes $P$ that are considerably simpler than the relatively simple ones that appear in the hardness proofs of Section 6. It should be noted in this connection that, with the exception of some small fixed values of $j$, even the problem of finding largest $j$-simplices in $n$-dimensional cubes offers many difficulties. For the full-dimensional case $(j=n)$ of that problem, the easiest subcase is that in which $n \equiv 3(\bmod 4)$. This easiest subcase subsumes the famous problem on the existence of Hadamard matrices. (See [HKL] for a discussion of the relationship of largest simplices to the Hadamard matrix problem and the Hadamard determinant problem; see also [GK4].)

For a few fixed small values of $n$ and $j$, simplex-maximizing algorithms of very low complexity have been found. Here are some references:

For $n=3$, Clarkson and Shor [CS] have an $O(m \log m)$ randomized algorithm that finds a largest 1-simplex in a 3-polytope with $m$ vertices. By "derandomizing" this algorithm, Chazelle et al. [CEGS] produced an $O\left(m^{1+\varepsilon}\right)$ deterministic procedure for the same purpose. That was improved to $O\left(m \log ^{3} m\right)$ by Brönniman et al. [BCM].

For $n=2$, the complexity of finding a largest 1-simplex or a largest 2-simplex in a convex polygon with $m$ vertices was shown by Dobkin and Snyder [DS] to be $O(m)$, assuming that $P$ 's $m$ vertices are presented in an order corresponding to traversal of $P$ 's boundary. (Finding such an order is an $O(m \log m)$ computation.)

\section{Hardness Results for $\mathscr{V}$-Polytopes}

The complexity results of this and the following section all establish the hardness of certain specifications of problems $\Pi_{1}-\Pi_{3}$. In each case the dimension $n$ is permitted to vary. This section treats $\mathscr{\mathscr { }}$-polytopes, and $\mathscr{H}$-polytopes appear in Section 6.

The hardness proofs of the present section are based, by way of a sequence of transformations, on the known hardness of detecting the presence of a Hamilton cycle (hereafter, $H$-cycle) in a directed graph (hereafter, digraph). This problem is strongly $\mathbb{N P}$-complete (see, e.g., [GJ]), and it appears in Karp's original list of NP-complete problems [K2]. One of the transformations uses a "graph gadget" of Papadimitriou and Steiglitz [PS], and the most crucial transformation (to a problem involving determinants) is due to Papadimitriou and Yannakakis [PY].

The main result of this section asserts that (the decision problem related to) finding a largest $n$-simplex in an $n$-dimensional $\mathscr{V}$-polytope is $\mathbb{N P}$-hard. Other hardness results are obtained from this by means of suitable geometric transformations. We could begin the proof by starting directly from the H-cycle problem for an arbitrary digraph. However, we start instead from a restriction of the H-cycle problem due to Plesnik, because that leads to a sharper form of the main result. Plesnik's result $[\mathrm{P}]$ implies that the hardness of the $\mathrm{H}$-cycle problem persists even 
when the input is restricted to planar digraphs in which, at each node, the indegree is at most 2 and the outdegree is at most 2 . We use the term $P$-digraph to refer to (not necessarily planar) digraphs satisfying this condition on degrees. (An alternative would be to add, to the requirement defining a P-digraph, the condition that each node has indegree 1 or outdegree 1 . The construction of $J^{\prime}$ from $J$ in the proof of Lemma 5.1, or of $G$ from $G_{0}$ in the proof of Theorem 5.2, makes it clear that hardness of the $\mathrm{H}$-cycle problem and the hardness results of Lemma 5.1 persist under this additional requirement.)

Recall that when $\psi$ and $\zeta$ are positive-valued functions with domain $\mathbb{N}$, the function $\psi$ is said to be of order $\Omega(\zeta)$, and we write $\psi(n)=\Omega(\zeta(n))$, provided a positive constant $\mu$ exists such that $\psi(n) \geq \mu \zeta(n)$ for all $n \in \mathbb{N}$.

Lemma 5.1. For each function $\psi: \mathbb{N} \rightarrow \mathbb{N}$, consider the following two problems:

$\mathrm{HAM}_{\psi}$ :

Instance: $\quad A$ planar $P$-digraph $D=(V, A)$ in which $|V|$ is prime and $|A| \leq|V|+$ $\psi(|V|)$.

Question: Does $D$ admit an $H$-cycle?

$\mathrm{UNIHAM}_{\psi}$ :

Instance: $\quad A$ P-digraph $D=(V, A)$ in which $|V|$ is prime and $|A| \leq|V|+\psi(|V|)$; the arc-set of an $H$-cycle $H$ in $D$.

Question: Does $D$ admit an $H$-cycle other than $H$ ?

If the function $\psi$ is bounded, then both problems are solvable in polynomial time. However, if $\psi(n)=\Omega\left(n^{1 / k}\right)$ for some $k \in \mathbb{N}$, then both problems are $\mathbb{N P}$-complete.

Proof. Let $n=|V|$. To prove the first assertion, note that if a digraph $D$ has $n$ nodes and at most $n+c$ arcs, then the number of ways of choosing a sequence of $n$ arcs of $D$ is $O\left(n^{c}\right)$. Each such choice can be tested in polynomial time to determine whether it corresponds to an H-cycle.

To establish the NPP-hardness of $\mathrm{HAM}_{\psi}$ when $\psi(n)=\Omega\left(n^{1 / k}\right)$, we produce a polynomial transformation of the $\mathrm{H}$-cycle problem for P-digraphs to $\mathrm{HAM}_{\psi}$. Starting from an arbitrary P-digraph $G$ with $r$ nodes and $s$ arcs, choose a node $v$ of $G$, add a new node $v^{\prime}$, replace each arc $(v, w)$ by an arc $\left(v^{\prime}, w\right)$, and add a new arc $\left(v, v^{\prime}\right)$. Denote the new digraph by $D_{1}$. Since $\left(v, v^{\prime}\right)$ is the only arc that starts at $v$ and the only arc that ends at $v^{\prime}$, it is clear that each $\mathrm{H}$-cycle in $D_{1}$ uses the arc $\left(v, v^{\prime}\right)$ and also that $G$ admits an $H$-cycle if and only if $D_{1}$ admits one. Now, for each $t \geq 2$, produce a digraph $D_{t}$ from $D_{1}$ by replacing the arc $\left(v, v^{\prime}\right)$ of $D_{1}$ by a path $P_{t}$ of length $t$ from $v$ to $v^{\prime}$. Clearly, $G$ admits an $\mathrm{H}$-cycle if and only if $D_{t}$ admits one. Note that the digraph $D_{t}$ has $r+t$ nodes and $s+t$ arcs, and that $D_{t}$ is itself a planar P-digraph for each $t$.

Saying that $\psi(n)=\Omega\left(n^{1 / k}\right)$ means that a constant $\mu>0$ exists such that $\psi(n) \geq \mu n^{1 / k}$ for all $n \in \mathbb{N}$. To complete the transformation, choose $t_{0} \in \mathbb{N}$ such that $t_{0} \geq\left(r^{2} / \mu\right)^{k}$. With $\mu$ known, this can be done in polynomial time. Then choose $t \geq t_{0}$ such that $r+t$ is prime. This too can be done in polynomial time, for there is 
a prime between $t_{0}+r$ and $2\left(t_{0}+r\right)$, and each integer in this interval can be tested for primality in time that is polynomial in terms of the original input data. Now, with $n=r+t, m=s+t$,

$$
(m-n)^{k}<r^{2 k} \leq \mu^{k} t<\mu^{k} n \leq \psi(n)^{k},
$$

whence

$$
m \leq n+\psi(n) .
$$

Since the digraph $D_{t}$ has $n$ nodes and $m$ arcs, $D_{t}$ does provide an instance of the problem $\mathrm{HAM}_{\psi}$. Hence that problem is $\mathbb{N P}$-hard.

The NP-hardness of UNIHAM ${ }_{4}$ is proved with the aid of a "graph gadget" used by Papadimitrious and Steiglitz in [PS]. This gadget is the digraph $G=(W, B)$, where

$$
W=\{w, x, p, q, y, z\}
$$

and

$$
B=\{(w, y),(w, z),(x, q),(p, w),(p, q),(q, p),(q, z),(y, x),(y, p),(z, x)\} .
$$

As was noted in [PS], $G$ has the following property: If $H$ is an H-cycle in a digraph $F$, and $G$ is embedded as a subdigraph of $F$ in such a way that

$$
\begin{aligned}
& \text { each arc of } F \backslash G \text { that ends in } G \text { ends at } w \text { or } y \text {, and } \\
& \text { each arc of } F \backslash G \text { that starts in } G \text { starts at } x \text { or } z \text {, }
\end{aligned}
$$

then the path $W=(w, y, p, q, z, x)$ or the path $Y=(y, x, q, p, w, z)$ is part of $H$.

Now let $J$ be a P-digraph that is given as input to the H-cycle problem for P-digraphs, and let $u_{1}, \ldots, u_{k}$ denote the nodes of $J$. For $1 \leq i \leq k$, add a new node $v_{i}$ and a new arc $\left(u_{i}, v_{i}\right)$, and replace each original $\operatorname{arc}\left(u_{i}, u_{j}\right)$ by a new $\operatorname{arc}\left(v_{i}, u_{j}\right)$. There is a natural one-to-one correspondence between H-cycles in $J$ and those in $J^{\prime}$. Note that in $J^{\prime}$, each node $u_{i}$ has outdegree 1 and indegree at most 2 , and each node $v_{i}$ has indegree 1 and outdegree at most 2 .

Let $G_{1}, \ldots, G_{k}$ be copies of $G$ that are vertex-disjoint from $J^{\prime}$ and from each other. In each of these copies, use the obvious subscript notation for the nodes and for the paths $W_{i}$ and $Y_{i}$ that correspond to $W$ and $Y$, respectively. Then form a digraph $J^{\prime \prime}$ by identifying (for $1 \leq i \leq k$ ) $w_{i}$ with $u_{i}$ and $x_{i}$ with $v_{i}$, and add arcs $\left(z_{1}, y_{2}\right), \ldots,\left(z_{k-1}, y_{k}\right),\left(z_{k}, y_{1}\right)$. Then the digraph $J^{\prime \prime}$ has the H-cycle given by

$$
y_{1}, Y_{1}, z_{1}, y_{2}, Y_{2}, z_{2}, y_{3}, \ldots, Y_{k}, y_{k}, y_{1},
$$

and this is the only $\mathrm{H}$-cycle in $J^{\prime \prime}$ that uses any of the $\operatorname{arcs}\left(z_{i}, y_{i+1}\right)$. It is easy to verify that the remaining $\mathrm{H}$-cycles of $J^{\prime \prime}$ (if there are any) are in one-to-one correspondence with the H-cycles of $J$. For example, if $u_{1}, \ldots, u_{k}, u_{1}$ happens to describe an H-cycle of $J$, then the corresponding $\mathrm{H}$-cycle of $J^{\prime \prime}$ is given by

$$
u_{1}=w_{1}, W_{1}, x_{1}=v_{1}, u_{2}=w_{2}, W_{2}, x_{2}=v_{2}, \ldots, W_{k}, x_{k}=v_{k}, u_{1} .
$$

It follows that the number of H-cycles of $J^{\prime \prime}$ is just one more than the number of H-cycles of $J$.

Note that the above digraph $J^{\prime \prime}$ is still a P-digraph, though it may not be planar. That completes the proof, except for the final technicality of replacing one of the 
$\operatorname{arcs}\left(z_{i}, y_{i+1}\right)$ by a path in such a way that the desired sparseness is obtained and the final number of nodes is prime.

The proof of the following result is essentially contained in an argument used by Papadimitriou and Yannakakis [PY] to establish the co-NP-completeness of the problem of deciding whether a given set of linear inequalities describes an integer polyhedron. Their main construction is a transformation from the general problem of detecting an $\mathrm{H}$-cycle in a digraph, under the special assumption that the digraph has a prime number of nodes. We repeat part of their proof, because our proof of Theorem 5.3 uses their construction.

Theorem 5.2. Suppose that the function $\psi: \mathbb{N} \rightarrow \mathbb{N}$ is monotone and that $\psi(n)=$ $\Omega\left(n^{1 / k}\right)$ for some $k \in \mathbb{N}$. Then the following problem $\mathrm{MAXDET}_{\psi}$ is strongly $\mathbb{N}$ P-complete:

Instance: $\quad n, \mu \in \mathbb{N}$, an $n \times m$ matrix $Q$ with $n \leq m \leq n+\psi(n)$ and with all entries in $\{-1,0,1\}$.

Question: Does $Q$ have an $n \times n$ submatrix $B$ such that $|\operatorname{det}(B)| \geq \mu$ ?

Proof. Let $G_{0}=\left(V_{0}, A_{0}\right)$ be a P-digraph regarded as input for the problem $\mathrm{HAM}_{\psi}$. A directed bipartite P-digraph $G=(V, A)$ is then constructed as follows:

- For each $v \in V_{0}$, add a new node $w=w(v)$ and set

$$
V=V_{0} \cup W_{0}=\left\{v: v \in V_{0}\right\} \cup\left\{w(v): v \in V_{0}\right\} .
$$

- Let $A^{\prime}=\left\{(v, w): v \in V_{0}, w=w(v)\right\}$, let $A^{\prime \prime}=\left\{\left(w\left(v_{1}\right), v_{2}\right):\left(v_{1}, v_{2}\right) \in A_{0}\right\}$, and set $A=A^{\prime} \cup A^{\prime \prime}$.

It is clear that $G$ admits an $\mathrm{H}$-cycle if and only if $G_{0}$ admits one. Set, as an abbreviation, $p=\left|V_{0}\right|, n=|V|=2 p$, and $m=|A|=\left|A_{0}\right|+p$. Let the points of $V_{0}$ be denoted by $v_{1}, \ldots, v_{p}$ and let those of $W_{0}$ be denoted by $w_{1}, \ldots, w_{p}$ with $w_{i}=w\left(v_{i}\right)$ for $1 \leq i \leq p$. Set $A^{\prime}=\left\{a_{1}, \ldots, a_{p}\right\}$ and $A^{\prime \prime}=\left\{a_{p+1}, \ldots, a_{m}\right\}$.

Now let $Q$ denote the $n \times m$ matrix that is obtained from $G$ 's node-arc incidence matrix by replacing the last row (the one that corresponds to $w_{p}$ ) by a row $(1, \ldots, 1,0, \ldots, 0)$ where the 1 -entries correspond precisely to the $\operatorname{arcs}$ in $A^{\prime}$. Thus, if $\hat{A}_{G}$ denotes the matrix that is obtained from $G$ 's node-arc incidence matrix by deleting the last row, $Q$ is of the following form:

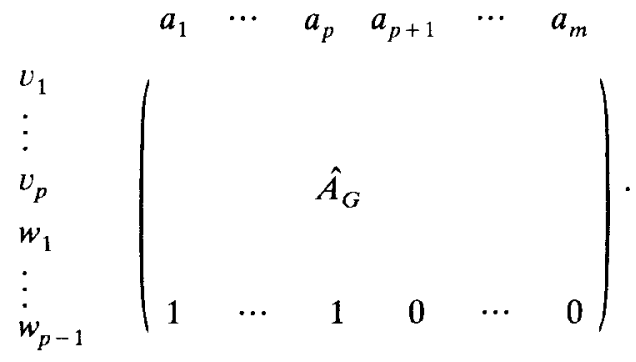


Papadimitriou and Yannakakis [PY] show that $Q$ contains an $n \times n$ submatrix $B_{0}$ with $\left|\operatorname{det}\left(B_{0}\right)\right| \geq p$ if and only if $G$ admits an H-cycle. Since, except for the last row, the matrix $Q$ is totally unimodular, it is easy to see that $|\operatorname{det}(B)| \leq p$ for all $n \times n$ submatrices $B$. Hence it follows that MAXDET, the problem of deciding whether a given $\{-1,0,+1\}$-matrix contains a maximum square submatrix whose determinant exceeds in absolute value a given bound, is strongly $\mathbb{N P}$-complete.

To complete the proof, refer to Theorem 5.1 and note that if, in the input digraph $\left(V_{0}, A_{0}\right),\left|A_{0}\right| \leq\left|V_{0}\right|+\psi\left(\left|V_{0}\right|\right)$, then

$$
m=\left|A_{0}\right|+\left|V_{0}\right| \leq 2\left|V_{0}\right|+\psi\left(\left|V_{0}\right|\right)=n+\psi\left(\frac{n}{2}\right) \leq n+\psi(n) .
$$

From the way in which $Q$ was formed from the node-arc incidence matrix of a digraph, it follows that no column or row of $Q$ has more than three nonzero entries. This sparseness, in addition to the fact that $Q$ is in a sense "almost square," is reflected in the structure of the polytope constructed from $Q$ in the proof of Theorem 5.3.

As a complement to Theorem 5.2, we note that [DGH] establishes the $\mathbb{N P}$ completeness (but not strong $\mathbb{N P}$-completeness) of the problem whose instance consists of an $n \times m$ integer matrix $A$ along with an integer $\lambda$, and whose question is whether there is an $n \times n$ submatrix of $A$ whose determinant has the value $\lambda$.

Theorem 5.3. The following problem, $\mathscr{V}-\mathrm{MAXSIMP}_{\psi}$ ( a variant of $\Pi_{1}$ ), is $\mathbb{N P}$-complete whenever the monotone function $\psi: \mathbb{N} \rightarrow \mathbb{N}$ is of order $\Omega\left(n^{1 / k}\right)$ for some $k \in \mathbb{N}$ :

Instance: $\quad n \in \mathbb{N}$, an $n$-dimensional $\mathscr{Y}$-polytope with vertex set $V$ such that $|V| \leq$ $n+\psi(n) ;$ a positive rational $\lambda$.

Question: Is there an $n$-simplex $S$ with vertices in $V$ such that $\operatorname{vol}(S) \geq \lambda$ ?

In fact the problem is $\mathbb{N} P$-complete under the additional restriction that $V \subset\{-1,0,1\}^{n}$.

Proof. Using a standard formula for simplex volume, it is easy to see (without any restrictions on $|V|$ that the problem belongs to the class $\mathbb{N P}$. To establish $\mathbb{N P}$. hardness, we extend the argument and construction of the preceding proof so as to

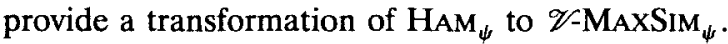

Let $Q$ denote the matrix of the preceding proof, and note first that all column vectors of $Q$ lie in a hyperplane. In fact, it follows from the construction of $G$ that $Q$ 's submatrix consisting of the entries in the first $p$ rows and columns is the $p \times p$ identity matrix, while the submatrix of $Q$ that consists of the entries of the first $p$ rows and the last $m-p$ columns has exactly one nonzero entry in each column, and this entry is -1 . Hence, when $z=(-1, \ldots,-1,0, \ldots, 0,2)^{T} \in \mathbb{R}^{n}$ with entries -1 as the first $p$ coordinates, and $Q=\left(q_{1}, \ldots, q_{m}\right)$, then

$$
\left\langle z, q_{1}\right\rangle=\cdots=\left\langle z, q_{m}\right\rangle=1
$$


Now suppose that $B=\left(q_{i_{1}}, \ldots, q_{i_{n}}\right)$ is an $n \times n$ submatrix of $Q$. Then

$$
|\operatorname{det}(B)|=n ! \operatorname{vol}_{n}\left(\operatorname{conv}\left\{0, q_{i_{1}}, \ldots, q_{i_{n}}\right\}\right)=\frac{(n-1) !}{\sqrt{p+2}} \operatorname{vol}_{n-1}\left(\operatorname{conv}\left\{q_{i_{1}}, \ldots, q_{i_{n}}\right\}\right)
$$

Let

$$
M=\left(\begin{array}{rrrrrrr}
1 & \cdots & 0 & 0 & \cdots & 0 & 0 \\
\vdots & \ddots & \vdots & \vdots & \ddots & \vdots & \vdots \\
0 & \cdots & 1 & 0 & \cdots & 0 & 0 \\
0 & \cdots & 0 & 1 & \cdots & 0 & 0 \\
\vdots & \ddots & \vdots & \vdots & \ddots & \vdots & \vdots \\
0 & \cdots & 0 & 0 & \cdots & 1 & 0 \\
-\frac{1}{2} & \cdots & -\frac{1}{2} & 0 & \cdots & 0 & 1
\end{array}\right)
$$

and let $q_{1}^{\prime}=M q_{1}, \ldots, q_{m}^{\prime}=M q_{m}$. Then the points $q_{i}^{\prime}$ all lie in the affine subspace $\mathbb{R}^{n-1} \times\left\{\frac{1}{2}\right\}$. Note further that $\operatorname{det}(M)=1$, and this implies that, with $B^{\prime}=$ $\left(q_{i_{1}}^{\prime}, \ldots, q_{i_{n}}^{\prime}\right)$,

$$
\begin{aligned}
|\operatorname{det}(B)| & =\left|\operatorname{det}\left(B^{\prime}\right)\right|=n ! \operatorname{vol}_{n}\left(\operatorname{conv}\left\{0, q_{i_{1}}^{\prime}, \ldots, q_{i_{n}}^{\prime}\right\}\right) \\
& =\frac{(n-1) !}{2} \operatorname{vol}_{n-1}\left(\operatorname{conv}\left\{q_{i_{1}}^{\prime}, \ldots, q_{i_{n}}^{\prime}\right\}\right)
\end{aligned}
$$

Hence, if $P^{\prime}=\operatorname{conv}\left\{q_{1}^{\prime}, \ldots, q_{m}^{\prime}\right\}$, then $Q$ has an $n \times n$ submatrix $B$ with $|\operatorname{det}(B)| \geq \mu$ if and only if the $(n-1)$-dimensional $\mathscr{T}$-polytope $P^{\prime}$ contains an $(n-1)$-simplex whose volume is at least

$$
\frac{2 \mu}{(n-1) !}
$$

Since the polytope $P^{\prime}$ is contained in the affine subspace $\mathbb{R}^{n-1} \times\left\{\frac{1}{2}\right\}$, it can be identified with a polytope $P$ in $\mathbb{R}^{n-1}$ which is given as the convex hull of those vectors that are obtained from the vectors $q_{1}^{\prime}, \ldots, q_{m}^{\prime}$ by deleting the last coordinate. Hence, a polynomial-time algorithm for $\mathscr{V}-\mathrm{MAXSiMP}_{\psi}$ would lead to a polynomialtime algorithm for the corresponding problem $\mathrm{MAxDET}_{\psi}$ and would eventually yield a polynomial-time algorithm for the problem $\mathrm{HAM}_{\psi}$. Finally, note that $-1,0$, and 1 are the only numbers that appear among the coordinates of the vertices of the constructed polytope $P$.

We now turn to the problem of finding a largest $j$-simplex in an $n$-dimensional $\mathscr{V}$-polytope when $j$ is permitted to be less than $n$. It is clear from Theorems 4.1 and 4.2 that in order to establish hardness, both $j$ and the excess of the number of vertices over $n$ must tend to $\infty$ with $n$. To extend the hardness result of Theorem 5.3, we need two lemmas. 
Lemma 5.4. With $\mathbb{R}^{n}=\mathbb{R}^{j} \times \mathbb{R}^{k}$, suppose that $P$ is a j-dimensional polytope in $\mathbb{R}^{j}$ and $B$ is an orthonormal basis for $\mathbb{R}^{k}$. Let $\rho$ and $R$ be respectively a lower bound on $P$ 's inradius and an upper bound on $P$ 's circumradius, with $0<\rho<1<R<\infty$. Suppose further that the origin 0 is an incenter of $P$, and that 0 is not an affine combination of fewer than $n+1$ vertices of $P$. For each $\varepsilon>0$, let $P_{\varepsilon}=\operatorname{conv}(P \cup \varepsilon B)$. If $\varepsilon \leq$ $(\rho / R)^{j} / j$, then each largest bound $j$-simplex $S$ in $P_{\varepsilon}$ is already contained in $P$.

Proof. Let $S$ denote a largest $j$-simplex bound to $P_{\varepsilon}$. (Recall that by Theorem 2.2 there is always a bound simplex among the largest.) Suppose that, for some $r \in\{1, \ldots, j+1\}$, the simplex $S$ contains precisely $r$ vertices that belong to the set $\varepsilon B$, while the remaining vertices belong to $P$.

Note first that the volume of a largest $j$-simplex $S_{0}$ in $P$ is at least as large as the volume of a regular $j$-simplex in a Euclidean $j$-ball of radius $\rho(C)$. Hence, by a standard formula,

$$
\operatorname{vol}\left(S_{0}\right) \geq \frac{(j+1)^{(j+1) / 2}}{j ! j^{j / 2}} \rho^{j} .
$$

By the same formula, together with the fact that regular simplices are the largest ones contained in a given ball,

$$
\operatorname{vol}\left(S_{k}\right) \leq \frac{(k+1)^{(k+1) / 2}}{k ! k^{k / 2}} R^{k}
$$

for each $k$-simplex $S_{k}$ in $P$ with $1 \leq k \leq j-1$.

Now suppose first that $2 \leq r \leq j$, and let $G$ denote the convex hull of those vertices of $S$ that belong to $P$. The vertices of $S$ in the set $\varepsilon B$ form a regular $(r-1)$-simplex $F$ of edge-length $\varepsilon \sqrt{2}$, whence a standard formula yields

$$
\operatorname{vol}(F)=\frac{\sqrt{r}}{(r-1) !} \varepsilon^{r-1}
$$

Since

$$
\operatorname{dist}(0, \operatorname{aff}(F))=\frac{\varepsilon}{\sqrt{r}} \quad \text { and } \operatorname{dist}(0, \operatorname{aff}(G)) \leq R,
$$

it follows with the aid of Lemma 1.5 and Theorem 1.6 that

$$
\begin{aligned}
\operatorname{vol}^{2}(S) & \leq \frac{1}{j !^{2}}\left(\frac{j-r+1}{j-r}\right)^{j-r}(j-r+1)\left(\varepsilon^{2}+\rho R^{2}\right) R^{2(j-r)} \varepsilon^{2(r-1)} \\
& \leq \frac{1}{j !^{2}} e(j-r+1) R^{2(j-r+1)} \varepsilon^{2(r-1)} \leq \frac{1}{j(j-1) !^{2}} e R^{2 j} \varepsilon^{2} \\
& \leq \frac{1}{j(j-1) !^{2}} e R^{2 j} \frac{1}{j}\left(\frac{\rho}{R}\right)^{2 j} \leq \frac{1}{j\left(j !^{2}\right)} e \rho^{2 j}
\end{aligned}
$$




$$
<\frac{(j+1)^{j+1}}{j !^{2} j^{j}} \rho^{2 j} \leq \operatorname{vol}^{2}\left(S_{0}\right)
$$

The case $r=j+1$ is even easier; in fact,

$$
\operatorname{vol}^{2}(S) \leq \frac{j+1}{j !^{2}} \varepsilon^{2 j}<\frac{(j+1)^{j+1}}{j !^{2} j^{j}} \rho^{2 j} \leq \operatorname{vol}^{2}\left(S_{0}\right),
$$

since $\varepsilon<\rho$.

Let us, finally, deal with the case $r=1$. Then $G$ is a facet of $S$. Let $H=\operatorname{aff}(G)$, let $H^{\prime}$ denote the supporting hyperplane of $\rho \mathbb{B}^{j}$ in $\mathbb{R}^{j}$ that does not separate $G$ from 0 , and let $c$ denote the point of support. Then the set $S^{\prime}=\operatorname{conv}(\{c\} \cup G)$ is a $j$-simplex in $P$, and

$$
\operatorname{vol}\left(S^{\prime}\right)=\frac{1}{j}(\operatorname{dist}(0, G)+\rho) \operatorname{vol}(G)
$$

Since $\varepsilon<\rho$, and since precisely one of the vertices of $S$ is in $\varepsilon B$, it follows with the aid of standard formulas that

$$
\operatorname{vol}^{2}(S)=\frac{(j-1) !}{j !}\left(\operatorname{dist}(\{0\}, G)^{2}+\varepsilon^{2}\right) \operatorname{vol}(G)<\operatorname{vol}^{2}\left(S^{\prime}\right) .
$$

That completes the proof of Theorem 5.4.

Lemma 5.5. With $\mathbb{R}^{n}=\mathbb{R}^{j} \times \mathbb{R}^{k}$, suppose that $P$ is a j-dimensional $\mathscr{T}$-polytope in $\mathbb{R}^{j}$ and that $\pi$ is an integer polynomial such that $k \leq \pi(j)$. Let $B, \varepsilon$, and $P_{\varepsilon}$ be as in Lemma 5.4. Then a rational $\mathscr{Y}$-presentation of $P_{\varepsilon}$ can be produced in time that is bounded by a polynomial in $L$, the size of $P$ 's $\mathscr{Y}$-presentation.

Proof. Just observe that a positive lower bound on $P$ 's inradius and a finite upper bound on $P$ 's circumradius can be computed in polynomial time (see, e.g., [GK3]), that the vertices of $P_{\varepsilon}$ are either vertices of $P$ or points of $\varepsilon B$, and that the increase in dimension from $j$ to $n$ in this construction, and hence also the increase in the number of vertices, is bounded by a polynomial in $j$.

Theorem 5.6. Suppose that the monotone function $\psi: \mathbb{N} \rightarrow \mathbb{N}$ and the function $\gamma$ : $\mathbb{N} \rightarrow \mathbb{N}$ are both of order $\Omega\left(n^{1 / k}\right)$ for some $k \in \mathbb{N}$, and that $1 \leq \gamma(n) \leq n$ for each $n \in \mathbb{N}$. Then the following version, $\Lambda_{1}$, of problem $\Pi_{1}$ is $\mathbb{N} P$-complete:

Instance: $\quad n \in \mathbb{N}$, an $n$-dimensional $\mathscr{V}$-polytope with vertex set $W$ such that $|W| \leq$ $n+\psi(n) ;$ a positive rational $\lambda$.

Question: Is there a $\gamma(n)$-simplex $S$ with vertices in $W$ such that $\operatorname{vol}^{2}(S) \geq \lambda$ ?

Proof. Membership in NP is obvious. The NP-hardness is established by means of a transformation from the problem $\mathscr{V}$-MAXSIMP ${ }_{\psi}$ of Theorem 5.3. In describing the 
transformation, we use $B_{j}^{r}$ to denote the set consisting of the last $r$ standard unit vectors of $\mathbb{R}^{r+j}$.

Let $(j, P, \lambda)$ be an instance of the problem $\mathscr{V}$-MAXSIMP ${ }_{\psi}$, and let $V$ denote $P$ 's vertex set. By our conditions there is a constant $\kappa \in \mathbb{N}$ such that

$$
\left(\frac{n}{\kappa}\right)^{1 / k} \leq \gamma(n) \leq n
$$

whence with $n=\kappa j^{k}$, we have

$$
0 \leq \gamma(n)-j \leq \kappa j^{k} .
$$

We now apply the construction of Lemmas 5.4 and 5.5 twice, using the notation of Lemma 5.4. First we replace the polytope $P=\operatorname{conv}(V)$ by the polytope

$$
P_{\varepsilon}=\operatorname{conv}\left(V \cup \varepsilon B_{j}^{\gamma(n)-j}\right) \subset \mathbb{R}^{\gamma(n)},
$$

and note that, by Lemma 5.4, $(j, P, \lambda)$ is a "yes" instance of $\mathscr{V}-\mathrm{MAXSIMP}_{\psi}$ if and only if $\left(\gamma(n), P_{\varepsilon}, \lambda\right)$ is a "yes" instance of $\mathscr{V} \mathrm{MAXSIMP}_{\psi}$. Then we raise the dimension by $n-\gamma(n)$, replacing $P_{\varepsilon}$ by the set

$$
Q_{\varepsilon}=\operatorname{conv}\left(P_{\varepsilon} \cup \varepsilon B_{\gamma(n)}^{n-\gamma(n)}\right)
$$

and noting that, by Lemma $5.4,\left(\gamma(n), P_{\varepsilon}, \lambda\right)$ is a "yes" instance of $\mathscr{V}$-MAXSIMP $\psi$ if and only if $\left(n, Q_{\varepsilon}, \lambda^{2}\right)$ is a "yes" instance of $\Lambda_{1}$.

Observe that $\varepsilon$ is bounded by a polynomial in $L$, that the increase in dimension is bounded by $\kappa j^{m}$, and that the increase in the number of vertices is therefore also bounded by $\kappa j^{m}$. From these facts, in conjunction with Lemma 5.5, it follows easily that the composite transformation from $\left(\gamma(n), P_{\varepsilon}, \lambda\right)$ to $\left(n, Q_{\varepsilon}, \lambda\right)$ runs in polynomial time. Hence, by Theorem 5.3, problem $\Lambda_{1}$ is $\mathbb{N P}$-hard.

Note that the conditions of Theorem 5.6 are satisfied when $\gamma(n)=\max \{1, n-\mu\}$ for a nonnegative integer constant $\mu$, and also when $\gamma(n)=\max \{1,\lfloor\mu n]\}$ for a fixed rational $\mu$ with $0<\mu \leq 1$.

The following theorem (along with Theorem 6.3 for $\mathscr{X}$-polytopes) is of interest in view of the uniqueness results concerning "typical" bodies and polytopes in Theorems $2.7-2.9$.

Theorem 5.7. If the functions $\psi$ and $\gamma$ satisfy the conditions of Theorem 5.6, then the following version $\Lambda_{3}$ of problem $\Pi_{2}$ is $\mathbb{N P}$-complete:

Instance: $\quad n \in \mathbb{N}$, an $n$-dimensional $\mathscr{Y}$-polytope $P$ with vertex set $V$ such that $|V| \leq$ $n+\psi(n) ;$ a largest $\gamma(n)$-simplex $S$ in $P$.

Question: Is there more than one largest $\gamma(n)$-simplex in $P$ ?

Proof. To see that $\Lambda_{3}$ belongs to the class NP, use the fact (from Theorem 2.2) 
that if there is more than one largest $\gamma(n)$-simplex in $P$, then there are at least two bound $\gamma(n)$-simplices that are largest. To establish $\mathbb{N P}$-hardness, use the second part of Lemma 5.1 and review the earlier transformations in this section, noting that each transformation is parsimonious in the sense of [J, p. 107]. To be specific:

- In Theorem 5.2's transformation from the digraph $G_{0}$ to a matrix $Q$, the number of $\mathrm{H}$-cycles in $G_{0}$ is equal to the number of $n \times n$ submatrices $B$ of $Q$ for which $|\operatorname{det}(B)| \geq p$.

- In Theorem 5.3's transformation from the matrix $Q$ to a polytope $P^{\prime}$, the number of $Q$ 's submatrices of the mentioned sort is equal to the number of bound $(n-1)$-simplices $S$ in the $(n-1)$-polytope $P^{\prime}$ for which $\operatorname{vol}(S) \geq$ $2 \mu /((n-1) !)$.

- In Theorem 5.6's transformation from problem $\mathscr{V}-\mathrm{MAXSIMP}_{\psi}$ to problem $\Lambda_{1}$, the number of full-dimensional simplices whose volume is at least as great as the specified threshold for $\mathscr{V}-\mathrm{MAXSIMP}_{\psi}$ is the same as the number of $\gamma(n)$ dimensional simplices whose volume is at least as great as the specified threshhold for $\Lambda_{1}$. (This can be used to show that the number of largest simplices of the specified dimensions is the same in the two cases.)

Hence the uniqueness question in Lemma 5.1 is transformed to the uniqueness question in Theorem 5.7, and since the former is $\mathbb{N P}$-hard so is the latter. (The parsimonious nature of these transformations can be used also to show that in each of Theorems 5.2, 5.3, 5.6, and 5.7, the associated counting problem is \#P-hard.)

Note that it follows from Theorem 5.7 that the corresponding version of $\Pi_{2}$ is also $\mathbb{N P}$-hard.

If an $n$-polytope $T$ is a simplex, the largest $j$-simplices in $T$ are the same as the largest $j$-faces of $T$. When $j$ or $n-j$ is bounded, the number of such $j$-faces is bounded by a polynomial in $n$ and hence a largest $j$-face can be found in polynomial time. However, for the case in which the dimension $j$ is "suitably intermediate" in terms of $n$, finding a largest $j$-face of an $n$-simplex becomes difficult. The next theorem makes this precise. Note that, for each fixed $k \in \mathbb{N}$ and each fixed rational $\mu$ with $0<\mu<1$, the theorem's conditions on $\gamma$ are satisfied if $\gamma(n)=\left\lceil\mu n^{1 / k}\right\rceil$ and also if $\gamma(n)=\left\lceil n-\mu n^{1 / k}\right\rceil$. In particular, they are satisfied if $\gamma(n)=\lceil\mu n\rceil$. However, we do not have any complexity results for the cases in which $\gamma(n)$ is $\lceil\log n\rceil$ or $\lceil n-\log n\rceil$.

Theorem 5.8. Suppose that the function $\gamma: \mathbb{N} \rightarrow \mathbb{N}$ satisfies the following conditions:

(i) For each $n \in \mathbb{N}, 1 \leq \gamma(n) \leq n$.

(ii) $\gamma(n)=\Omega\left(n^{1 / m}\right)$ for some $m \in \mathbb{N}$.

(iii) A monotone function $\psi: \mathbb{N} \rightarrow \mathbb{N}$ and a $k \in \mathbb{N}$ exist such that

(1) $\psi(n)=\Omega\left(n^{1 / k}\right)$,

(2) $\gamma(n+\psi(n)) \leq n$ for all $n \in \mathbb{N}$.

Then the following version of problem $\mathrm{II}_{1}$ is $\mathbb{N P}$-complete:

Instance: $\quad n \in \mathbb{N}$, an $n$-dimensional $\mathscr{V}$ or $\mathscr{K}$-simplex $T$ in $\mathbb{R}^{n}$, a positive rational $\lambda$. Question: Is there a $\gamma(n)$-face $S$ of $T$ such that $\operatorname{vol}^{2}(S) \geq \lambda$ ? 
Proof. The proof of hardness is accomplished by a transformation of problem $\Lambda_{1}$ of Theorem 5.6. Let $j \in \mathbb{N}, n(j)=j+\psi(j)$, and define the function $\gamma^{\prime}: \mathbb{N} \rightarrow \mathbb{N}$ by $\gamma^{\prime}(j)=\gamma(n(j))$. Now consider an arbitrary instance $(j, P, \lambda)$ of $\Lambda_{1}$ with parameter functions $\psi$ and $\gamma^{\prime}$. Note that, by (i) and (iii(2)), $1 \leq \gamma^{\prime}(j) \leq j$ for all $j \in \mathbb{N}$, and this fact, in conjunction with properties (ii) and (iii(1)), assures that $\psi$ and $\gamma^{\prime}$ are proper parameter functions for $\Lambda_{1}$.

Let $V=\left\{v_{0}, \ldots, v_{n}\right\}$ denote $P$ 's vertex set, and let $e_{1}, \ldots, e_{n}$ be the standard unit vectors of $\mathbb{R}^{n}$. We may assume without loss of generality that $P$ is an integer $\mathscr{V}$-polytope, whence (by Theorem 1.4) the squares of the volumes of any two bound simplices in $P$ are either the same or differ at least by $(n !)^{-2}$.

Now set

$$
v_{i}^{\prime}=v_{i} \quad \text { for } \quad i=0, \ldots, j \quad \text { and } \quad v_{i}^{\prime}=v_{i}+\varepsilon e_{i} \quad \text { for } \quad i=j+1, \ldots, n \text {, }
$$

where $0 \leq \varepsilon \leq 1$. Then the set

$$
T_{\varepsilon}=\operatorname{conv}\left\{v_{0}^{\prime}, \ldots, v_{n}^{\prime}\right\}
$$

is an $n$-simplex in $\mathbb{R}^{n}$. Note that there is a one-to-one correspondence between the bound simplices in $P$ and the faces of $T_{\varepsilon}$ of dimension at most $j$. (In our notation, the latter carries the subscript $\varepsilon$, the former the subscript 0 .) Let $S_{\varepsilon}$ denote a $\gamma^{\prime}(j)$-face of $T_{\varepsilon}$. Suppose without loss of generality that 0 is a vertex of $S_{\varepsilon}$, and let $v_{i_{1}}^{\prime}, \ldots, v_{i_{j}}^{\prime}$ denote the other vertices of $S_{\varepsilon}$. If $A_{\varepsilon}$ is the matrix with rows $v_{i_{1}}^{\prime T}, \ldots, v_{i_{1}}^{\prime T}$, then by Theorem 1.4,

$$
\left(\gamma^{\prime}(j) !\right)^{2} \operatorname{vol}^{2}\left(S_{\varepsilon}\right)=\operatorname{det}\left(A_{\varepsilon} A_{\varepsilon}^{T}\right)
$$

Note that $\operatorname{det}\left(A_{\varepsilon} A_{\varepsilon}^{T}\right)$ is a polynomial in $\varepsilon$ of degree at most $2(n-j)$, with constant term $\left(\gamma^{\prime}(j) !\right)^{2} \operatorname{vol}^{2}\left(S_{0}\right)$. The remaining terms are bounded from above by $\varepsilon$ times the sum of the absolute values of all subdeterminants of $A_{0} A_{0}^{T}$ of arbitrary dimension, and this sum is bounded above by $2^{2(j+L)}$, a number whose size is polynomial in the size $L$ of the presentation of $T$. Hence, when $\varepsilon=2^{-2(j+L)^{-1}} /(n !)$,

$$
\operatorname{vol}\left(S_{\varepsilon}\right) \leq \lambda \quad \text { if and only if } \operatorname{vol}\left(S_{0}\right) \leq \lambda
$$

This shows that $(j, P, \lambda)$ is a "yes" instance of $\Lambda_{1}$ if and only if the simplex $T_{\varepsilon}$ contains a $\gamma^{\prime}(j)$-face whose squared volume is at least $\lambda$. Since $\gamma(n)=\gamma^{\prime}(j)$, that completes the proof of Theorem 5.8.

\section{Hardness Results for $\mathscr{H}$-Polytopes}

It turns out that in $\mathscr{H}$-polytopes of variable dimension, even finding largest $j$ simplices of fixed low dimension $j$ is $\mathbb{N} P$-hard. This contrast to the $\mathscr{V}$-presented case (see Theorem 4.1) arises from the fact that for $\mathscr{X}$-polytopes the number of vertices 
can increase exponentially in terms of the size of the presentation. Unfortunately, we have not succeeded in establishing hardness for the problem of finding largest full-dimensional simplices in $\mathscr{Z}$-polytopes. (However, see the conjecture near the end of Section 4.)

In dealing with $\mathscr{H}$-polytopes, our tool for proving $\mathbb{N} P$-hardness is the fact that the following problem $[0,1]$ PARMAX and some of its close relatives are $\mathbb{N} P$-hard.

Instance: $\quad n \in \mathbb{N}$, linearly independent rational vectors $x_{1}, \ldots, x_{n} \in \mathbb{R}^{n}$; a positive rational $\beta$.

Question: Is the maximum of \|\|$^{2}$ on the parallelotope $\sum_{i=1}^{n}[0,1] x_{i}$ greater than or equal to $\beta$ ?

Although the parallelotope here is not explicitly $\mathscr{H}$-presented, the given presentation can be used to produce (in polynomial time) a rational $\mathscr{H}$-presentation of size $\pi(L)$, where $\pi$ is a fixed polynomial and $L$ is the size of the original presentation. It then follows from an observation of Khachiyan [K3] that max $\|c\|: c \in C\} \leq 4^{\pi(L)}$. That fact is used below in the proof of Theorem 6.2.

The NP-hardness of $[0,1]$ PARMAX was established in [BGKV] by transformation from the problem Not-ALL-EQUAL-3SAT of Schaefer [S1]. (See [GK1] for a different transformation serving the same purpose.) [0,1]PARMAX was later used in [GK2] and [GK3] to establish the hardness of other problems involving parallelotopes, including the special cases of problems $\Gamma_{1}$ and $\Gamma_{2}$ of Theorem 6.2 in which $\gamma(n) \equiv 1$. ([GK3] is concerned with $j$-balls, but when $j=1$ the $j$-balls and the $j$-simplices are the same.)

Hardness results for certain problems are often used to produce hardness proofs for other problems. To facilitate such use in a polytopal setting, it is desirable to work with polytopes that are as simple as possible. The hardness proofs in this section use polytopes that are convex hulls of simplices and parallelotopes. We require a lemma to prepare for the hardness proofs.

Lemma 6.1. With $\mathbb{R}^{n}=\mathbb{R}^{j} \times \mathbb{R}^{k}$, suppose that $B$ is an orthonormal basis for $\mathbb{R}^{j}$ and $C$ is $a$ body in $\mathbb{R}^{k}$. Let $\rho=\max \{\|x\|: x \in C\}$, and for each $\mu>0$ let $C_{\mu}=$ conv $(\mu B \cup C)$. If $\mu \geq 3 j \rho$, then for each $j$-simplex $S$ in $C_{\mu}$ it is true that

$$
(j !)^{2} \operatorname{vol}^{2}(S) \leq\left(\mu^{2}+j \rho^{2}\right) \mu^{2 j-2},
$$

with equality if and only if $S=\operatorname{conv}(\mu B \cup\{c\})$ for some $c \in C$ with $\|c\|=\rho$.

Proof. (The assumption that $\mu \geq 3 j \rho$ can be weakened, but it is adequate for our purposes.)

For $0 \leq r \leq j$, let us say that a $j$-simplex $S$ in $C_{\mu}$ is of type $r$ if $r+1$ vertices of $S$ are those of an $r$-simplex $G$ in $C$ and the remaining $j-r$ vertices of $S$ belong to the set $\mu B$ and hence are those of a regular $(j-r-1)$-simplex $F$ of edge-length $\mu \sqrt{2}$. (Use appropriate conventions for the special cases in which $r \in\{0, j-1, j\}$.) By Theorem 2.2, there is a largest $j$-simplex in $C_{\mu}$ that is of type $r$ for some $r$, and we want to estimate the volumes of the various types. Since (with appropriate 
conventions in the mentioned special cases) the standard formulas yield

$$
\operatorname{vol}(F)=\frac{\sqrt{j-r}}{(j-r-1) !} \mu^{j-r-1}, \quad \operatorname{vol}(G) \leq \frac{(r+1)^{(r+1) / 2}}{r ! r^{r / 2}} \rho^{r}
$$

and

$$
\operatorname{dist}(0, \operatorname{aff}(F))=\frac{\mu}{\sqrt{j-r}}, \quad \operatorname{dist}(0, \operatorname{aff}(G)) \leq \rho
$$

it follows with the aid of Lemma 1.5 and Theorem 1.6 that

$$
(j !)^{2} \operatorname{vol}^{2}(S) \leq \varphi(r),
$$

where

$$
\begin{aligned}
& \varphi(0)=\left(\mu^{2}+j \rho^{2}\right) \mu^{2 j-2}, \\
& \varphi(j)=\left(\frac{j+1}{j}\right)^{j}(j+1) \rho^{2 j},
\end{aligned}
$$

and

$$
\varphi(r)=\left(\frac{r+1}{r}\right)^{r}(r+1)\left(\frac{\rho}{\mu}\right)^{2 r}\left(\mu^{2}+(j-r) \rho^{2}\right) \mu^{2 j-2}, \quad 1 \leq r \leq j-1 .
$$

It follows also that when $r=0$, the upper bound $\varphi(0)$ is attained if and only if $S=\operatorname{conv}(\mu B \cup\{c\})$ for some $c \in C$ with $\|c\|=\rho$.

With

$$
\xi(\tau)=\left(\frac{\tau+1}{\tau}\right)^{\tau}
$$

we have $\xi(\tau)<e$ for all $\tau>0$. Hence if $\mu / \rho>3 j$, then

$$
\frac{\varphi(0)}{\varphi(r)}=\frac{1}{\xi(r)(r+1)} \frac{\mu^{2}+j \rho^{2}}{\mu^{2}+(j-r) \rho^{2}}\left(\frac{\mu}{\rho}\right)^{2 r}>\frac{1}{j e}\left(\frac{\mu}{\rho}\right)^{2 r} \geq \frac{4}{e} 2^{2 r-2} j^{2 r-1}>1
$$

when $1 \leq r \leq j-1$, while

$$
\frac{\varphi(0)}{\varphi(j)}>\frac{1}{(j+1) e} j^{2 j}>1
$$

That completes the proof.

Note that conditions (i) and (ii) in the following theorem are satisfied when the function $\gamma$ is constant, and also when $\gamma(n)=\lfloor\mu n\rfloor$ for a fixed rational $\mu$ with $0<\mu<1$. (See the growth conditions in Theorems 5.6-5.8). 
Theorem 6.2. Suppose that the function $\gamma: \mathbb{N} \rightarrow \mathbb{N}$ satisfies the following conditions:

(i) For each $n \in \mathbb{N}, 1 \leq \gamma(n) \leq n$.

(ii) There is a function $f: \mathbb{N} \rightarrow \mathbb{N}$, computable in polynomial time, such that, for each $k \in \mathbb{N}, f(k)-\gamma(f(k))=k$.

Then the following version, $\Gamma_{1}$, of problem $\Pi_{1}$ is $\mathbb{N P}$-complete and the following version, $\Gamma_{2}$, of problem $\Pi_{2}$ is $\mathbb{N P}$-hard:

Instance for $\Gamma_{1}: \quad n \in \mathbb{N}$, an n-dimensional $\mathscr{H}$-polytope $P \subset \mathbb{R}^{n}$, a positive rational $\lambda$. Question for $\Gamma_{1}$ : Does $P$ contain a $\gamma(n)$-simplex $S$ such that $\operatorname{vol}^{2}(S) \geq \lambda$ ?

Instance for $\Gamma_{2}: \quad n \in \mathbb{N}$, an $n$-dimensional $\mathscr{H}$-polytope $P \subset \mathbb{R}^{n}$.

Question for $\Gamma_{2}$ : Is the largest $\gamma(n)$-simplex in $P$ unique?

Proof. It follows from Theorem 2.2 that attention may in both cases be confined to bound simplices. It is then evident that problem $\Gamma_{1}$ belongs to the class $\mathbb{N P}$. The proofs of $\mathbb{N P}$-hardness will involve transformations from the problem [0,1]PARMAX.

Let $\left(k, \beta ; x_{1}, \ldots, x_{k}\right)$ be an instance of the problem [0,1]PARMAx, let $L$ denote the size of this instance, and let the polynomial $\pi$ be the one mentioned after the description of [0,1]PARMAX earlier in this section. Let $n=f(k), j=f(k)-k$, and $\mu=3 j 4^{\pi(L)}$. Form the $n$-polytope $C_{\mu}$ as in Lemma 6.1 , with the $k$-parallelotope $\sum_{i=1}^{n}[0,1] x_{i}$ playing the role of Lemma 6.1 's $k$-body $C$.

It is not hard to verify that the facets of $C_{\mu}$ are precisely the sets of the form $\operatorname{conv}(F \cup \mu B)$ where $F$ is a facet of $C$, and the sets of the form $\operatorname{conv}\left(C \cup \mu B^{\prime}\right)$ where $B^{\prime}$ consists of all but one point of the set $B$. Using this fact, it is easy in polynomial time to use the rational $\mathscr{H}$-presentation of $C$ in $\mathbb{R}^{k}$ to produce a rational $\mathscr{Z}$-presentation of $C_{\mu}$ in the containing space $\mathbb{R}^{n}=\mathbb{R}^{j} \times \mathbb{R}^{k}$. Now it is clear from Lemma 6.1 that the norm on $\mathbb{R}^{k}$ attains its maximum at a point $w$ of $C$ if and only if the set $S=\operatorname{conv}(\{w\} \cup \mu B)$ is a largest $j$-simplex in $C_{\mu}$. Hence the hardness of $\Gamma_{1}$ follows from that of $[0,1]$ PARMAX.

To deal with $\Gamma_{2}$, we use the same construction but now consider the $k$-parallelotope $C=\sum_{i=1}^{n}[0,1] x_{i} \subset \mathbb{R}^{k}$ as an instance of the problem UNIPARMAX from [GK2]. That problem's question is whether the maximum of the Euclidean norm is attained at more than one vertex of $C$. We do not expect either UNIPARMAX or $\Gamma_{2}$ to belong to the class $\mathbb{N P}$. However, UNIPARMAX is $\mathbb{N P}$-hard, as was established in [GK2] by using the NP-hardness of [0,1]PARMAX. From Lemma 6.1 it is clear that the norm on $\mathbb{R}^{k}$ attains its maximum at more than one point of the parallelotope $C$ if and only if the body $C_{\mu}$ contains more than one largest $j$-simplex. Hence we have a polynomial-time transformation of problem UNIPARMAX to problem $\Gamma_{2}$, and the NP-hardness of the latter problem follows from that of the former.

A different way of stating Theorem 6.2 is to say that whenever a function $\kappa: \mathbb{N} \rightarrow \mathbb{N}$ is bounded above by a polynomial in $n$, then it is $\mathbb{N} P$-hard, for a given $\mathscr{H}$-polytope $P$ in $\mathbb{R}^{n+\kappa(n)}$, to find a largest $\kappa(n)$-simplex in $P$ and it is also $\mathbb{N} P$-hard to decide whether the largest $\kappa(n)$-simplex in $P$ is unique. 


\section{Additional Comments and Problems}

\section{A. Two Problems Concerning Largest and c-Largest Simplices}

For an arbitrary $n$-body $B$, let $\rho(B)$ denote the ratio of $B$ 's volume to the volume of a largest $n$-simplex in $B$. When $B$ has a center of symmetry $c$, let $\rho_{0}(B)$ denote the ratio of $B$ 's volume to the volume of a $c$-largest $n$-simplex in $B$. Let $\mathbb{B}$ denote the $n$-dimensional Euclidean unit ball. It was proved by McKinney [M2] that $\rho_{0}(B) \geq$ $\rho_{0}(\mathbb{B})$ for all centrally symmetric $n$-bodies $B$, with equality if and only if $B$ is an ellipsoid. For $n \leq 3$ it had been proved earlier by Blaschke (see [BR]) that $\rho(B) \geq$ $\rho(\mathbb{B})$ for all $n$-bodies, with equality characterizing ellipses and ellipsoids at least in the case of smooth bodies. Macbeath [M1] extended the inequality to an arbitrary $n$, but as far as we know, the characterization result of Blaschke has not been extended beyond the three-dimensional case.

For a centrally symmetric $n$-body $C$, let $f(C)=\rho(C) / \rho_{0}(C)$. What is the range of $f(C)$ as $C$ ranges over centrally symmetric $n$-bodies, and for which $n$-bodies are the extreme values attained? The evaluation of $f(Q)$ for an $n$-cube $Q$ would also be of great interest. However, that is probably a very difficult problem, for as $n \rightarrow \infty$ both $\rho(Q)$ and $\rho_{0}(Q)$ become very difficult to evaluate precisely (see [HKL]).

\section{B. Close-to-Largest Simplices}

Since determination of a largest $n$-simplex in a given $n$-polytope $P$ seems to be difficult, the question of approximation becomes relevant. Some results can be easily derived by approximating the Löwner-John ellipsoid (the smallest ellipsoid containing $P$ ) and then using the facts that this ellipsoid is the affine image of the unit $n$-ball, that every largest simplex in the ball is regular, and that volume ratios are invariant under nonsingular affine transformations. This yields an approximation error that is similar to the error of volume approximation discussed in [GLS] (see [GK5]).

Other authors pursue (at least implicitly) a "weak Löwner-John simplex" approach, producing an $n$-simplex that is contained in a given polytope $P$ such that an appropriately dilated simplex contains $P$ or "almost contains" $P$. However, in terms of the volumes of the produced versus the largest simplices in $P$, the approximation error may again be exponential (see [AK], [DF], [FGK], and [W].)

Finally, note that once a "large" $n$-simplex $S$ in $P$ is produced, it is natural to try to take a largest $j$-faces of $S$ as an approximation of a largest $j$-simplex in $P$. Theorem 5.8 places, however, severe algorithmic limitations on such an approach.

\section{Possible Sharpening of Hardness Results}

The most significant gap in our hardness results is that they do not address the difficulty of finding a largest full-dimensional simplex in an $\mathscr{K}$-polytope. However, as 
is indicated in the conjecture following the proof of Theorem 4.3, we believe that this problem is also $\mathbb{N P}$-hard.

Section 5's hardness results for $\mathscr{T}$-polytopes involve lower bounds on the growth of the number $n+\psi(n)$ of vertices of the $n$-dimensional $\mathscr{Y}$-polytope $P$ under consideration, and also on the growth of the dimension $\gamma(n)$ of the simplices in $P$ whose volume is to be maximized. It would be interesting to know how far these growth conditions can be weakened. In particular, can they be replaced by logarithmic growth conditions?

Although the polytopes used in the hardness proofs of Sections 5 and 6 are not very complicated, it would be interesting to know whether they can be further simplified. In particular, does the hardness of $\mathscr{V}-\mathrm{MAXSIMP}_{\psi}$ in Theorem 5.3 persist for polytopes in which all vertex coordinates belong to $\{0,1\}$ ? (In the theorem as it stands, the coordinates are restricted to $\{-1,0,1\}$.)

In problem $\Gamma_{1}$ of Section 6 , does the hardness persist when the input polytope is required to be a parallelotope? (There is no chance of that for $\Gamma_{2}$, since when $j>1$ and $S$ is a largest $j$-simplex in a given centrally symmetric body, the reflection of $S$ in $P$ 's center is a largest $j$-simplex that is different from $S$.)

\section{Smallest Containing Simplices}

Much of this paper has been motivated by the difficult problem of finding a largest $n$-simplex contained in a given $n$-polytope $P$. Of equal interest, and perhaps of even greater algorithmic difficulty, is the problem of finding a smallest $n$-simplex $S$ containing $P$. A theorem of [K5] asserts that, for each smallest $S$, the centroid of each facet of $S$ belongs to $P$, but this information must be augmented by additional geometric conditions on $S$ in order to obtain a reasonable algorithm for actually finding a smallest containing simplex. For finding a smallest containing triangle (when $n=2$ ), the main algorithmic contributions are those of [KL], [OAMB], and [MC], [CM]. The last two papers establish a strong relationship between the smallest triangles containing a given convex polygon and the largest triangles contained in the polygon. The problem of finding a smallest tetrahedron containing a given 3-polytope has been studied in [VY].

\section{Acknowledgments}

We are indebted to P. Gruber, J. Matoušek, and J. O'Rourke for useful references.

\section{References}

[AK] D. Applegate and R. Kannan, Sampling and integration of near log-concave functions, Proc. 23rd ACM Symp. on Theory of Computing, 1990, pp. 156-163.

[BR] W. Blaschke and K. Reidemeister, Vorlesungen über Differentialgeometrie II. Affine Differentialgeometrie, Springer-Verlag, Berlin, 1923.

[B] L. M. Blumenthal, Distance Geometry, Oxford University Press, London, 1953. 
[BGKV] H. Bodlaender, P. Gritzmann, V. Klee, and J. Van Leeuwen, Computational complexity of norm-maximization, Combinatorica 10 (1990), 203-225.

[BCM] H. Brönniman, B. Chazelle, and J. Matoušek, Product range spaces, sensitive sampling and derandomization, Proc. 34th IEEE Symp. on Foundations of Computer Science, 1993, pp. $400-409$.

[CM] S. Chandran and D. M. Mount, A parallel algorithm for enclosed and enclosing triangles, Internat. J. Comput. Geom. Appl. 2 (1992), 191-214.

[CEGS] B. Chazelle, H. Edelsbrunner, L. Guibas, and M. Sharir, Diameter, width, closest line pair, and parametric searching, Discrete Comput. Geom. 10 (1993), 183-196.

[CS] K. Clarkson and P. Shor, Applications of random sampling to computational geometry. II, Discrete Comput. Geom. 4 (1989), 387-421.

[DS] D. P. Dobkin and L. Snyder, On a general method for maximizing and minimizing among certain geometric problems, Proc. 20th IEEE Symp. on Foundations of Computer Science, 1983, pp. 9-17.

[DF] M. E. Dyer and A. M. Frieze, Computing the volume of convex bodies: a case where randomness provably helps. In Probabilistic Combinatorics and Its Applications (B. Bollobás, ed.), Proceedings of Symposia in Applied Mathematics, Vol. 44, American Mathematical Society, Providence, RI, 1991, pp. 123-169.

[DGH] M. Dyer, P. Gritzmann, and A. Hufnagel, On the complexity of computing mixed volumes, Manuscript (1994).

[FGK] U. Faigle, N. Gademann, and W. Kern, A random polynomial time algorithm for wellrounding convex bodies, Discrete Math. (to appear).

[F] L. Fejes Tóth, Regular Figures, Pergamon Press, Oxford, 1964.

[GJ] M. Garey and D. S. Johnson, Computers and Intractability. A Guide to the Theory of NP-Completeness, Freeman, San Francisco, CA, 1979.

[GK1] P. Gritzmann and V. Klee, On the 0-1 maximization of positive definite quadratic forms, Operations Research Proceedings 1988, Springer-Verlag, Berlin, 1989, pp. 222-227.

[GK2] P. Gritzmann and V. Klee, Deciding uniqueness in norm-maximization, Math. Programming 57 (1992), 203-214.

[GK3] P. Gritzmann and V. Klee, Computational complexity of inner and outer $j$-radii of convex polytopes, Math. Programming 59 (1993), 163-213.

[GK4] P. Gritzmann and V. Klee, On the complexity of some basic problems in computational convexity: I. Containment problems, Discrete Math. 136 (1994), 129-174. Reprinted in: New Trends in Discrete Mathematics (W. Deuber, H.-J. Prömel, and B. Voigt, eds.), North-Holland, Amsterdam, 1995, to appear.

[GK5] P. Gritzmann and V. Klee, On the complexity of some basic problems in computational convexity: II. Volume and mixed volumes. In: Polytopes: Abstract, Convex and Computational (T. Bisztriczky, P. McMullen, R. Schneider, and A. Ivic Weiss, eds.), Kluwer, Boston, 1994. pp. 373-466.

[GLS] M. Grötschel, L. Lovász, and A. Schrijver, Geometric Algorithms and Combinatorial Optimization, Springer-Verlag, Berlin, 1988.

[G1] P. Gruber, Die meisten konvexen Körper sind glatt, aber nicht zu glatt, Math. Ann. 229 (1977), 259-266.

[G2] B. Grünbaum (with V. Klee, M. A. Perles, and G. C. Shephard), Convex Polytopes, Wiley-Interscience, London, 1967.

[HKL] M. Hudelson, V. Klee, and D. G. Larman, Largest $j$-simplices in $d$-cubes: Some relatives of the Hadamard maximum determinant problem, Manuscript, 1995.

[J] D. S. Johnson, A catalog of complexity classes. In: Handbook of Theoretical Computer Science. Vol. A (J. van Leeuwen, ed.), Elsevier and M.I.T. Press, Amsterdam and Cambridge, MA, 1990, pp. 67-161.

[K1] N. Karmarkar, A new polynomial-time algorithm for linear programming, Combinatorica 4 (1984), 373-397.

[K2] R. Karp, Reducibility among combinatorial problems. In: Complexity of Computer Computations (R. E. Miller and J. W. Thatcher, eds.), Plenum, New York, 1972, pp. 85-103.

[K3] L. G. Khachiyan, Polynomial algorithms in linear programming, U.S.S.R. Comput. Math. and Math. Phys. 20 (1980), 53-72. 
[K4] V. Klee, Some new results on smoothness and rotundity in normed linear spaces, Math. Ann. 139 (1959), 51-63.

[K5] V. Klee, Facet centroids and volume minimization, Studia Sci. Math. Hungar. 21 (1986), $143-147$

[KL] V. Klee and M. C. Laskowski, Finding the smallest triangle containing a given convex polygon, J. Algorithms 6 (1985), 359-375.

[L] N. Linial, Hard enumeration problems in geometry and combinatorics, SIAM J. Algebraic Discrete Methods 7 (1986), 331-335.

[M1] A. M. Macbeath, An external property of the hypersphere, Math. Proc. Cambridge Philos. Soc. 47 (1951), 245-247.

[M2] J. R. McKinney, On maximal simplices in a central convex set, Mathematika 21 (1974), $38-44$

[M3] P. McMullen, The maximum number of faces of a convex polytope, Mathematika 17 (1970), 179-184.

[MC] D. M. Mount and S. Chandran, A unified approach for finding enclosing and enclosed triangles, Proc. Allerton Conf. on Communications, Control, and Computing, 1988, pp. $87-96$.

[OAMB] J. O'Rourke, A. Aggarwal, S. Maddila, and M. Baldwin, An optimal algorithm for finding minimal enclosing triangles, J. Algorithms 7 (1986), 258-269.

[PS] C. H. Papadimitriou and K. Steiglitz, Some complexity results for the traveling salesman problem, Proc. 8th ACM Symp. on Theory of Computing, 1976, pp. 1-9.

[PY] C. H. Papadimitriou and M. Yannakakis, On recognizing integer polyhedra, Combinatorica 10 (1990), 107-109.

[P] J. Plesnik, The NP-completeness of the Hamiltonian cycle problem in planar digraphs with degree bound two, Inform. Process. Lett. 8 (1979), 291-297.

[S1] J. Schaefer, The complexity of satisfiability problems, Proc. 10th Ann. ACM Symp. on Theory of Computing, 1978, pp. 216-226.

[S2] D. Slepian, The content of some extreme simplexes, Pacific J. Math. 31 (1969), 795-808.

[S3] D. M. Y. Sommerville, An Introduction to the Geometry of $N$ Dimensions, Methuen, London, 1929 (reprinted in 1958 by Dover, New York).

[VY] G. Vegter and C. Yap, Minimal circumscribing simplices, Proc. 3rd Canadian Conf. on Computational Geometry, Vancouver, 1991, pp. 58-91.

[W] E. Weidner, A short note on Löwner-John simplices and volume approximation, Manuscript, 1994.

Received January 13, 1994, and in revised form August 19, 1994. 